\title{
BCG vaccination reduces the mortality of Mycobacterium tuberculosis-infected type 2 diabetes mellitus mice
}

\author{
Rajesh Kumar Radhakrishnan, Ramya Sivangala Thandi, Deepak Tripathi, Padmaja Paidipally, \\ Madeline Kay McAllister, Sachin Mulik, Buka Samten, and Ramakrishna Vankayalapati \\ Department of Pulmonary Immunology, Center for Biomedical Research, University of Texas Health Science Center at \\ Tyler, Texas, USA.
}

\begin{abstract}
Diabetes is a significant risk factor for the development of active tuberculosis. In this study, we used a mouse model of type 2 diabetes mellitus (T2DM) to determine the effect of prior Bacillus Calmette-Guérin (BCC) vaccination on immune responses to Mycobacterium tuberculosis (Mtb) infection. We found that, at 6-7 months after Mtb infection, $90 \%$ of the Mtb-infected T2DM mice died, whereas only $50 \%$ of BCG-vaccinated T2DM-Mtb-infected mice died. Moreover, $40 \%$ of the PBS-treated uninfected T2DM mice and $30 \%$ of the uninfected BCG-vaccinated T2DM mice died, whereas all uninfected and infected nondiabetic mice survived. BCG vaccination was less effective in reducing the lung bacterial burden of $M t b$-infected T2DM mice compared with $M t b$-infected nondiabetic mice. BCG vaccination significantly reduced lung inflammation in Mtb-infected T2DM mice compared with that of unvaccinated T2DM mice infected with Mtb. Furthermore, reduced mortality of BCG-vaccinated Mtb-infected T2DM mice is associated with expansion of IL-13-producing CXCR3+ Tregs in the lungs of Mtb-infected T2DM mice. Recombinant IL-13 and Tregs from BCG-vaccinated Mtb-infected T2DM mice converted proinflammatory M1 macrophages to antiinflammatory M2 macrophages. Our findings suggest a potentially novel role for BCC in preventing excess inflammation and mortality in T2DM mice infected with Mtb.
\end{abstract}

Conflict of interest: The authors have declared that no conflict of interest exists.

Copyright: ( 2020 , American Society for Clinical Investigation.

Submitted: September 25, 2019 Accepted: January 30, 2020 Published: March 12, 2020.

Reference information: /CI Insight. 2020;5(5):e133788

https://doi.org/10.1172/jici. insight.133788.

\section{Introduction}

Tuberculosis (TB), caused by Mycobacterium tuberculosis $(M t b)$, is a leading cause of death worldwide $(1,2)$. In 2017, 10 million people globally developed active TB disease, and 1.3 million deaths occurred as a result of TB disease $(2,3)$. It is estimated that one-third of the world's population has latent TB infection (LTBI), and $90 \%$ of these individuals remain healthy (4). However, approximately $10 \%$ of $\mathrm{LTBI}^{+}$individuals develop active disease during their lifetime $(5,6)$. Immunocompromised conditions such as malnutrition, aging, diabetes, smoking, and alcohol abuse, as well as coinfections with HIV and helminths, can enhance the risk of developing active TB in $\mathrm{LTBI}^{+}$individuals $(2,7-9)$.

Diabetes is one of the risk factors for active TB development. In 2017, it was estimated that 425 million of people worldwide were confirmed as diabetic or prediabetic (10), suggesting that it is important to understand the immune responses to $M t b$ in patients with diabetes. Type 2 diabetes mellitus (T2DM) patients with LTBI have a three-fold higher risk of developing active TB $(2,11,12)$, and poor glycemic control is a major risk factor for developing active TB (13). Hyperglycemia negatively regulates the innate and adaptive immune systems and subsequently leads to impaired defense against active TB (7, 14). Excess Th1- and Th17-mediated inflammatory responses were noted in TB patients with T2DM (15), and pathological immune responses in $M t b$-infected T2DM mice lead to enhanced mortality $(8,16)$. There is limited information available about the immune responses to $M t b$ in T2DM hosts $(11,15)$.

Bacillus Calmette-Guérin (BCG) is a live-attenuated strain of Mycobacterium bovis and has been used as a vaccine against the TB for almost a century (17-23). BCG immunization also has protective effects against viral infections (22); noninfectious diseases (24-27), such as hypertension-induced myocardial hypertrophy and cardiac fibrosis (28); and bladder and urothelial cancers $(27,29)$. Furthermore, BCG is known to reverse the advanced type 1 diabetes $(23,30)$. However, there is no information available about vaccine-induced protective immune responses against infections, including TB, in T2DM hosts. 
In the current study, we investigated the effects of prior BCG vaccination on the immune responses and survival of T2DM mice infected with $M t b$. We also determined BCG-induced immunoregulatory mechanisms that enhance the survival of $M t b$-infected T2DM mice.

\section{Results}

$B C G$ vaccination enhances the survival of Mtb-infected T2DM mice. We determined the effect of prior BCG vaccination on the mortality of T2DM mice infected with $M t b$. A schematic representation of BCG vaccination, development of T2DM, and Mtb infection is shown in Figure 1A. At the 6- to 7-month after infection (p.i.) time point, $90 \%$ of the $M t b$-infected T2DM mice died, whereas only $50 \%$ of BCG-vaccinated T2DM- $M t b-$ infected mice died $(P<0.01)$. Moreover, $40 \%$ of PBS-treated uninfected T2DM mice and 30\% of uninfected BCG-vaccinated T2DM mice died $(P<0.05)$, whereas all uninfected and infected nondiabetic mice survived (Figure 1B). Our findings demonstrate that BCG vaccination prevents the deaths of not only Mtb-infected T2DM mice, but also uninfected T2DM mice.

We measured the body weights and levels of blood glucose, serum insulin, triglyceride, free fatty acid, and cholesterol levels of all groups of mice at regular intervals until 330 days. As shown in Figure 1C, all nondiabetic (control and $M t b$-infected) mice gained significant weight compared with that of T2DM (control and Mtb-infected) mice. Blood glucose levels of T2DM mice (control and infected) were $\geq 250 \mathrm{mg} / \mathrm{dL}$ during the entire study period and were $80-125 \mathrm{mg} / \mathrm{dL}$ in all nondiabetic mice (Figure 1D). The serum insulin, triglyceride, and free fatty acid levels of all groups of T2DM mice increased and maintained significantly higher levels during the entire study period compared with those of all groups of nondiabetic mice (Figure 1, E-G). One month after infection, there was no significant difference in the serum cholesterol levels in all the PBS-treated T2DM and BCG-vaccinated T2DM mice (control and infected), but cholesterol levels were significantly decreased at 4 and 6 months in infected T2DM mice (unvaccinated and vaccinated) as compared with the levels in uninfected mice $(P<0.001)$ (Figure $1 \mathrm{H})$.

$B C G$ vaccination reduces bacterial burden less efficiently in T2DM mice during chronic Mtb infection. We asked whether the enhanced survival of BCG-vaccinated $M t b$-infected T2DM mice was due to a reduced lung bacterial burden in $M t b$-infected mice. One month after infection, BCG vaccination reduced lung bacterial burden by close to 2 logs in $M t b$-challenged nondiabetic mice compared with that of PBS-treated mice $(4.024 \pm 0.08$ versus $5.992 \pm 0.09 ; P<0.001)$ and 1.48 logs in the lungs of BCG-vaccinated T2DM mice compared with that of PBS-treated T2DM mice ( $4.668 \pm 0.16$ versus $6.146 \pm 0.08 ; P<0.001)$. Four months p.i., BCG vaccination reduced the lung bacterial burden of nondiabetic mice by $1.2 \operatorname{logs}(4.968 \pm 0.15$ versus $6.2 \pm 0.08 ; P<0.001$, Figure $2 \mathrm{~A}$ ) and 0.3 logs in T2DM mice compared with that of PBS-treated control mice $(5.892 \pm 0.14$ versus $6.2 \pm 0.08)$. A similar reduction in bacterial burden was noted in the spleen and liver (Figure 2, B and C). Our findings demonstrate that, in addition to marginally reducing bacterial burden, BCG vaccination protects $M t b$-infected T2DM mice through other mechanisms.

$B C G$ vaccination reduces immunopathology in the lungs of T2DM mice during chronic infection. Histopathological analysis of lung sections among the 8 groups of mice (control, T2DM, BCG-vaccinated control, BCG-vaccinated T2DM, infected control, infected T2DM, infected BCG-vaccinated control, and infected BCG-vaccinated T2DM) showed significantly more inflammation in T2DM mice infected with $M t b$ when compared with that of PBS-treated mice infected with $M t b(P<0.001)$. BCG-vaccinated Mtb-infected nondiabetic mice and BCG-vaccinated T2DM mice infected with $M t b$ displayed significantly reduced inflammation when compared with those of $M t b$-infected nondiabetic mice or $M t b$-infected T2DM mice at 4 months p.i. $(P<0.01)$ (Figure 2, D-G).

$B C G$ vaccination alters cytokine and chemokine levels in T2DM mice infected with $M t b$. In lung homogenates of mice in the 8 groups, we measured various cytokine and chemokine levels using multiplex (36-plex) ELISA kits. As previously published (8), the levels of pro- and antiinflammatory cytokines and chemokines were significantly elevated in $M t b$-infected T2DM mice at 1 and 4 months p.i. when compared with the levels in uninfected T2DM and infected nondiabetic mice (Figure 3 and Supplemental Figures 1 and 2; supplemental material available online with this article; https://doi.org/10.1172/jci.insight.133788DS1). BCG vaccination of T2DM mice infected with $M t b$ significantly reduced some of the pro- and antiinflammatory cytokines (IFN- $\alpha$, IL-1 $\alpha$, IL-1 $\beta$, IFN- $\gamma$, TNF- $\alpha$, IL-6, IL-3, IL-18, IL-23, IL-27, IL-9, and IL-31) and chemokines (GM-CSF, ENA-78, M-CSF, MCP-1, and MIP-1 $\beta$ ) but enhanced the levels of IL-13, IL-15, IP-10, and MIP-2 compared with the levels in nonvaccinated T2DM mice infected with Mtb (Figure 3 and Supplemental Figures 1 and 2). 
A PBS or Subcutaneous (s.c.) BCG Vaccination $\left(1 \times 10^{6} \mathrm{CFU}\right)$

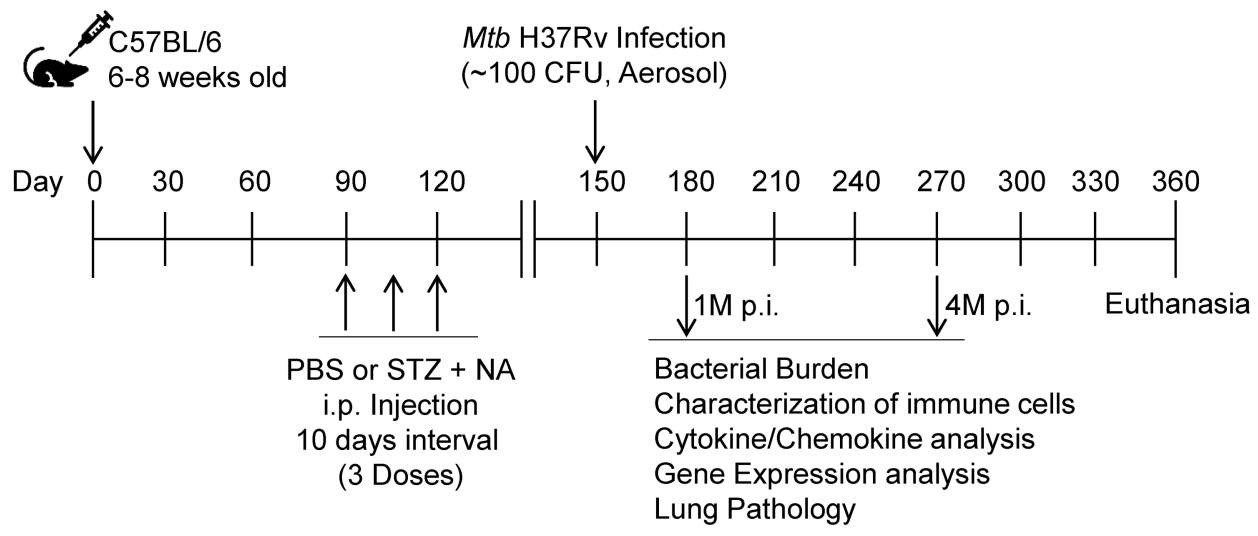

B
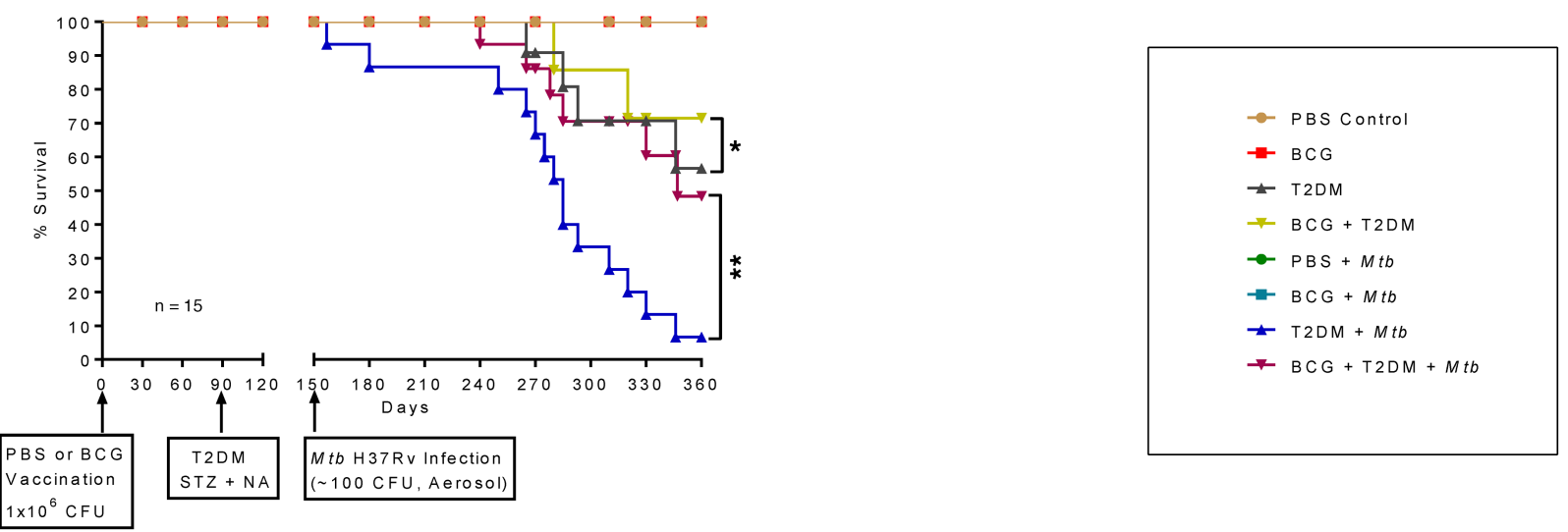

C

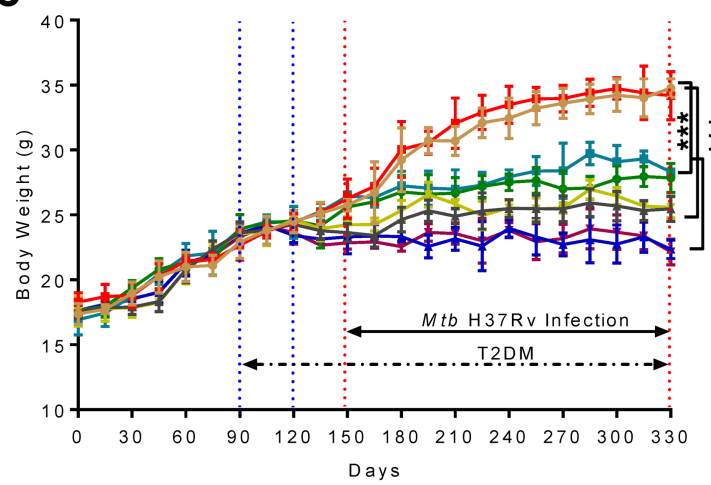

Days

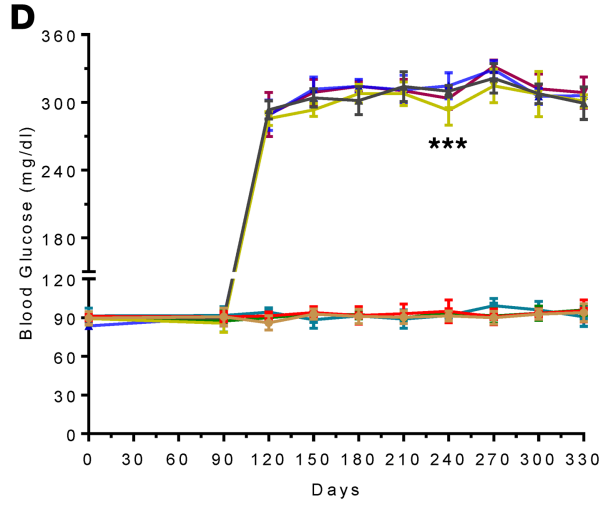

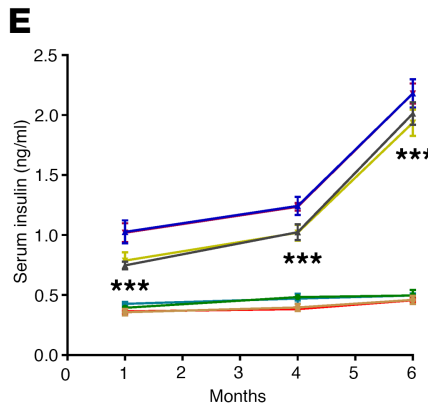
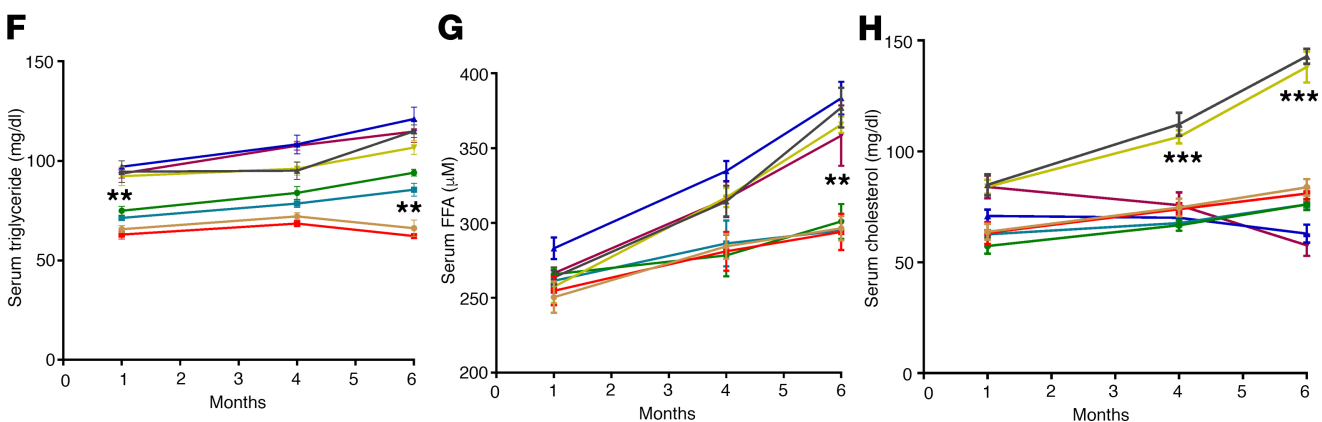

Figure 1. BCG vaccination enhances the survival of $\mathbf{M t b}$-infected T2DM mice. (A) A schematic representation of BCG vaccination, T2DM induction, and Mtb H37Rv infection is shown. Six- to 8-week-old C57BL/6 mice (15 mice per group) were given $100 \mu \mathrm{L}$ of phosphate-buffered saline (PBS; unvaccinated) or vaccinated s.c. with $1 \times 10^{6}$ CFU of BCG in $100 \mu \mathrm{L}$ of PBS. Three months after vaccination, T2DM was induced in some of the mice by the i.p. injection of streptozotocin (180 mg/kg body weight) and nicotinamide (60 mg/kg body weight), as described in Methods. PBS control, BCG-vaccinated, T2DM, or 
BCG-vaccinated T2DM mice were infected with 100 CFU of aerosolized Mtb. (B) The $P$ value for percent survival was calculated using the log rank test. The Kaplan-Meier survival curves of mice are shown. Data were pooled from 2 independent experiments $(n=10$ mice in 1 experiment; $n=5$ mice in another experiment). (C) Mouse body weight changes were determined every 15 days. (D) Random blood glucose levels were determined at monthly intervals for up to 10 months. (E-H) One, 4, and 6 months after Mtb infection, the serum insulin (E), triglyceride (F), free fatty acid (G), and cholesterol (H) levels were estimated. Experiments were performed 2 times, and each time, 2-3 mice per group were used (C-H). The data are shown as mean \pm SDs of $n=5$ mice per group. The statistical analysis was performed by 1-way ANOVA, followed by Tukey's multiple comparisons test. ${ }^{*} P<0.05,{ }^{* *} P<0.01$, and ${ }^{* * *} P<0.001$.

BCG-vaccinated Mtb-infected T2DM mouse lungs have reduced Th1, Th2, and Th17 cytokine-producing CD4 cells and increased Tregs and M2 macrophages. We characterized various immune cell populations in the lungs of control and infected mice in the 8 groups by flow cytometry. As shown in Figure 4 and Supplemental Table 1, one month after infection, there was a significant increase in Th1, Th2, Th17, and $\mathrm{CD}^{+} \mathrm{CD} 25^{+} \mathrm{Foxp}^{+}$cell populations in the lungs of BCG-vaccinated T2DM mice infected with $M t b$ compared with those of all other groups (Figure 4, A-D). Four months after infection, there was a significant decrease in the total number of Th1, Th2, and Th17 cytokine-producing $\mathrm{CD} 4^{+}$cell populations in the lungs of BCG-vaccinated T2DM mice infected with Mtb compared with that of unvaccinated (PBS-treated) Mtb-infected T2DM mice (Figure 4, A-C, and Supplemental Figures 3-8). In contrast, there was a significant increase in Tregs in $M t b$-infected BCG-vaccinated T2DM mice when compared with other groups of mice (Figure 4D and Supplemental Figures 6 and 10). As shown in Figure 4, G and $\mathrm{H}$, and Supplemental Figure 9, A and B, 4 months after infection, there were significantly higher numbers of $\mathrm{M} 1\left(\mathrm{~F} 4 / 80^{+} \mathrm{CD} 80^{+}\right)$macrophages in BCG-vaccinated nondiabetic mice and $\mathrm{M} 2\left(\mathrm{~F} 4 / 80^{+} \mathrm{CD} 206^{+}\right)$ macrophages in BCG-vaccinated diabetic mouse lungs. We found that Tregs in BCG-vaccinated T2DM mice infected with $M t b$ were found in proximity to the M2 macrophage phenotype (Supplemental Figure 9C). As shown in Supplemental Figure 9D, we found significantly higher expression of M2 macrophage gene markers (Arg1, CD163, and CD206) in BCG-vaccinated T2DM mouse lungs infected with Mtb compared with that of vaccinated nondiabetic mice infected with $M t b$.

$B C G$ vaccination expands $C X C R 3^{+}$Tregs in Mtb-infected T2DM mice. We isolated $\mathrm{CD} 4^{+} \mathrm{CD} 25^{+} \mathrm{Foxp} 3^{+}$cells from the above 8 groups of mice 4 months after $M t b$ infection and performed quantitative PCR (qPCR) analysis for various molecules expressed by Tregs. As shown in Figure 5A, Tregs from BCG-vaccinated Mtb-infected T2DM mice expressed significantly higher levels of CXCR3 compared with Tregs from other groups of mice. We also found significantly higher IL-13 mRNA expression by Tregs from BCG-vaccinated T2DM mice infected with Mtb compared with that of Tregs from other groups of mice (Figure 5A). We further phenotypically characterized the lung Treg population of the above-mentioned groups of mice and found significantly increased CXCR3 expression in Tregs from BCG-vaccinated T2DM mice infected with $M t b$ by flow cytometry (Figure 5B and Supplemental Figure 11) and confocal microscopy (Figure 5C).

T2DM had no effect on the immunosuppressive capacity of Tregs from BCG-vaccinated T2DM mice infected with $M t b$. We compared the immunosuppressive potential of Tregs from various groups of mice. Tregs were isolated from the 8 groups of mice 4 months after infection, as described in the Methods section. Splenocytes from T2DM mice were isolated, and Tregs were subsequently depleted. Treg-depleted splenocytes were labeled with CFSE and cocultured with Tregs from different groups (8 groups) of mice, as mentioned in the Methods section and as shown in Figure 6A. T2DM significantly inhibited the immunosuppressive capacity of Tregs (control and Mtb-infected mice) compared with that of Tregs from the remaining groups of mice (Figure 6B). BCG vaccination restored the immunosuppressive capacity of Tregs from uninfected $(P<0.05)$ and $M t b$-infected T2DM mice $(P<0.001)$ when compared with that of unvaccinated (uninfected) T2DM and $M t b$-infected T2DM mice (Figure 6B). In the above-mentioned cultured cells, we also measured various cytokine and chemokine levels using multiplex (36-plex) ELISA kits. As shown in Figure 6, C-E, and Supplemental Figure 12, Tregs from $M t b$-infected and uninfected T2DM mice were unable to inhibit IFN- $\gamma$, TNF- $\alpha$, and IL- 6 production by T2DM mouse splenocytes. These results demonstrate that BCG vaccination preserves the immunosuppressive function of Tregs in T2DM mice.

Tregs convert proinflammatory M1 macrophages to antiinflammatory M2 macrophages through IL-13R signaling. In Figure 5A, we found that Tregs from BCG-vaccinated T2DM mice infected with Mtb express higher levels of IL-13 mRNA when compared with the levels in all other groups of mice. We confirmed this by confocal microscopy (Figure 7A). We also cultured spleen cells or Treg-depleted spleen cells from BCG-vaccinated T2DM mice with $\gamma$-irradiated Mtb H37Rv $(\gamma M t b)$ and determined the M2 macrophage phenotype and IL-13 levels in culture supernatants. Treg-depleted spleen cells stimulated with $\gamma M t b$ were unable to expand 

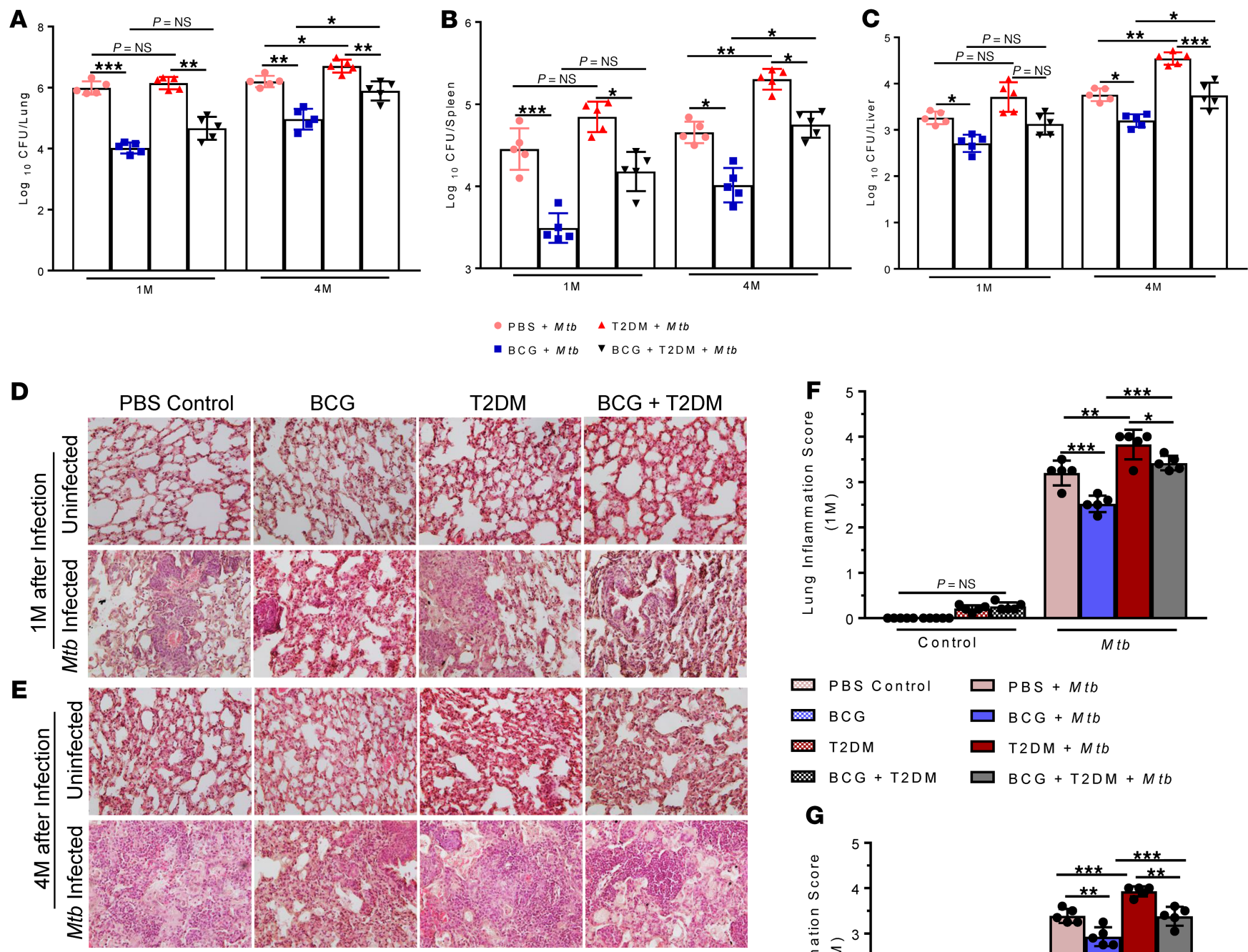

- $\mathrm{PBS}+M t b \quad \triangle \mathrm{T} 2 \mathrm{DM}+M t b$

- $\mathrm{BCG}+M t b \quad \boldsymbol{\nabla} \mathrm{BCG}+\mathrm{T} 2 \mathrm{DM}+M t b$
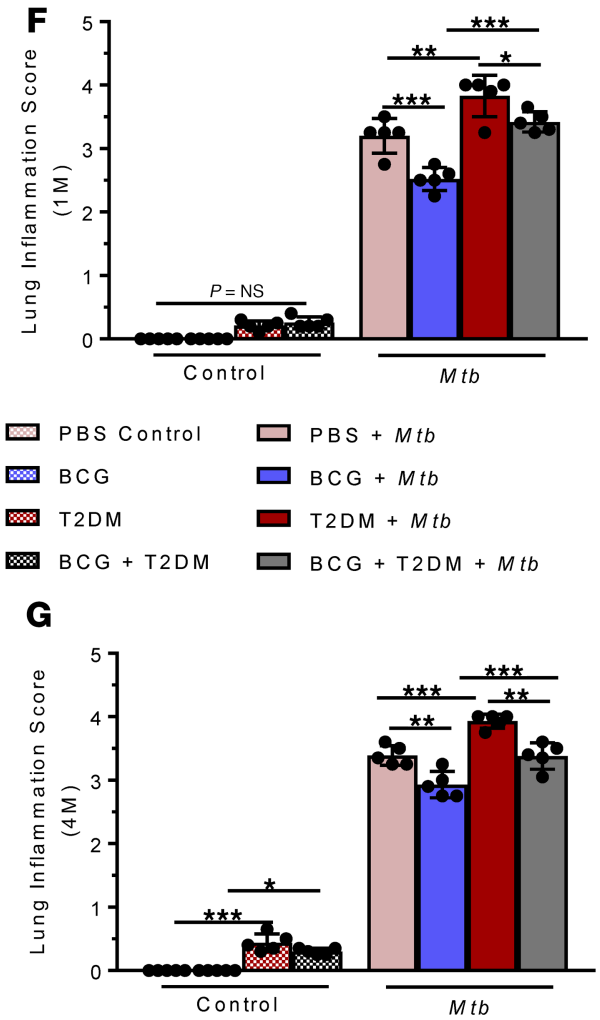

Figure 2. BCG vaccination reduces bacterial burden less efficiently in T2DM mice during chronic infection. PBS control, BCG-vaccinated, T2DM, or BCG-vaccinated T2DM mice were infected with the Mtb as described in Figure 1. (A-C) One and 4 months after Mtb infection, the bacterial burden in the lungs (A), spleen (B), and liver (C) was measured. Experiments were performed 2 times, and each time, 2-3 mice per group were used. The data are shown as mean \pm SDs of $n=5$ mice per group. The statistical analysis was performed by 1-way ANOVA, followed by Tukey's multiple comparisons test. ${ }^{*} P<0.05$, ${ }^{* *} P<0.01$, and ${ }^{* *} P<0.001$. At 1 and 4 months after infection (p.i.), lungs from uninfected or Mtb-infected mice were isolated and formalin fixed. Paraffin-embedded tissue sections were prepared, and H\&E staining was performed. Inflamed lung areas were compared between the groups. ( $\mathbf{D}$ and $\mathbf{E})$ Representative bright-field microscopic histology images were taken from multiple fields at $20 \times$ objective lens. (F and $\mathbf{G}$ ). The lung inflammation score was assessed at 1 and 4 months p.i. Experiments were performed 2 times, and each time, 2-3 mice per group were used. The data are shown as mean \pm SDs of $n=5$ mice per group. The statistical analysis was performed by 1-way ANOVA, followed by Tukey's multiple comparisons test. ${ }^{*} P<0.05,{ }^{* *} P<0.01$, and ${ }^{* * *} P<0.001$.

the M2 macrophages (CD45+F4/80+Arg1 ${ }^{+}$cells) (Figure 7B and Supplemental Figure 13, A-C). As shown in Figure 7B, depletion of Tregs significantly reduced IL-13 levels in the culture supernatants, suggesting that Tregs are the major source of IL-13 in BCG-vaccinated T2DM mice.

IL-13 is primarily produced by Th2 cells, but other cell types - including Tregs — also produce IL-13 during activation (31-33). IL-13 inhibits proinflammatory cytokines and chemokines and acts as an important antiinflammatory molecule $(31,33)$. The antiinflammatory Salmonella vaccine CFA/I protects mice from experimental autoimmune encephalomyelitis (EAE) by IL-13-producing Tregs (32). We next asked whether the expansion of M2 macrophages in BCG-vaccinated T2DM mice depends on IL-13 and 
A

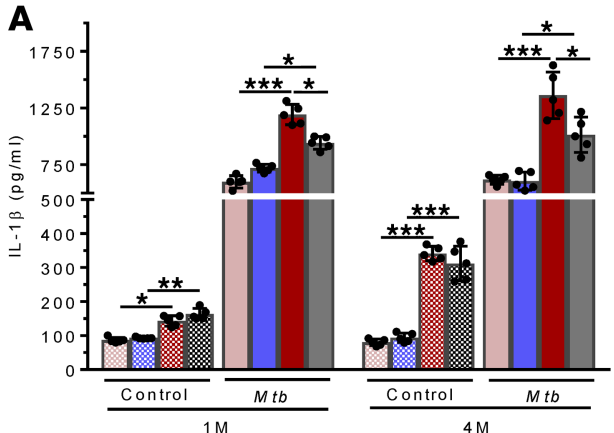

B

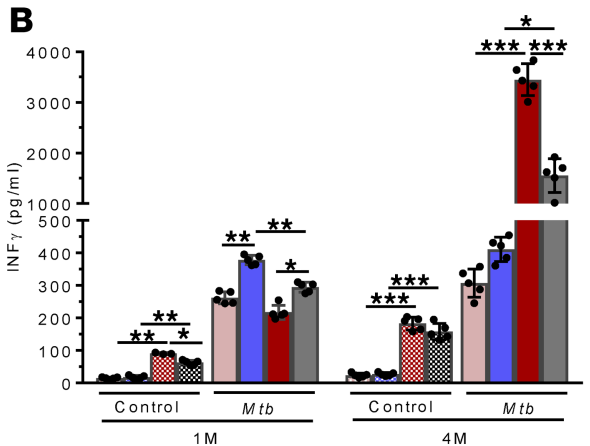

D

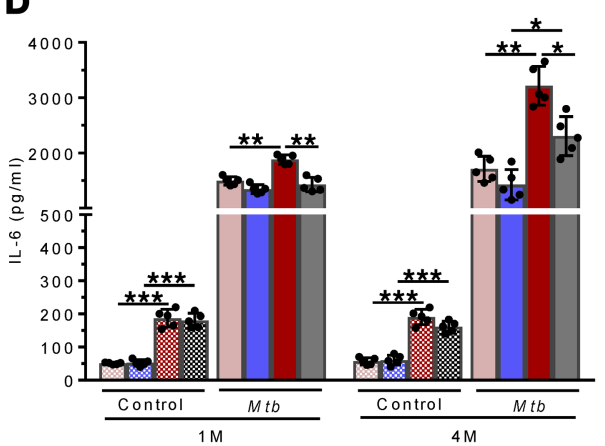

$\mathbf{F}$

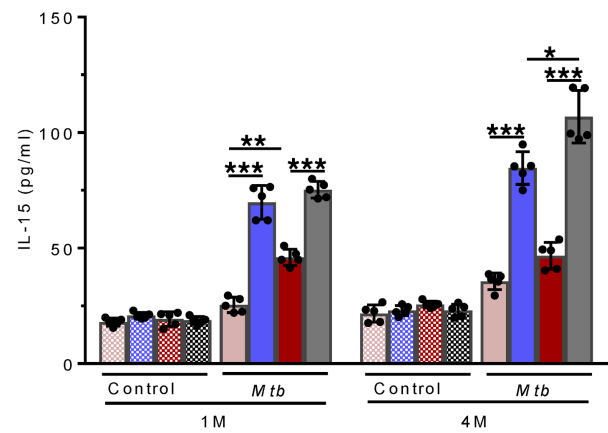

H

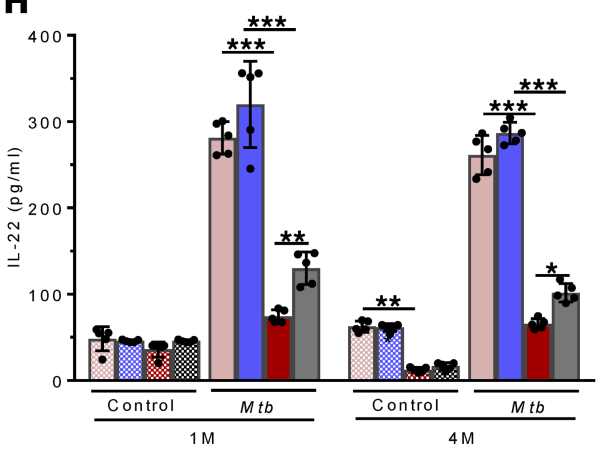

$\square$ PBS Control

BCG

T2DM

$B C G+T 2 D M$

$\square \mathrm{PBS}+M t b$

$\square B C G+M t b$

$\square T 2 D M+M t b$

$\square B C G+T 2 D M+M t b$

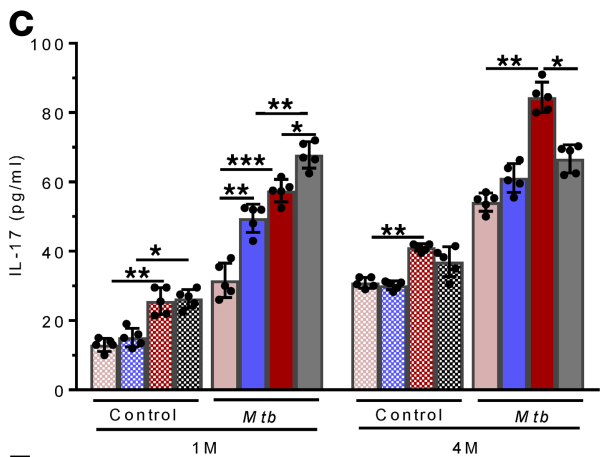

E

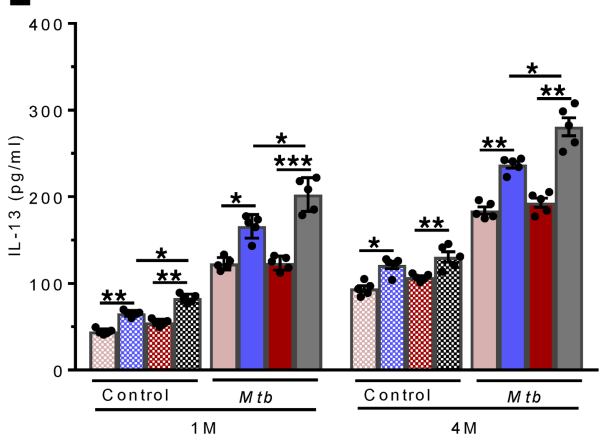

G

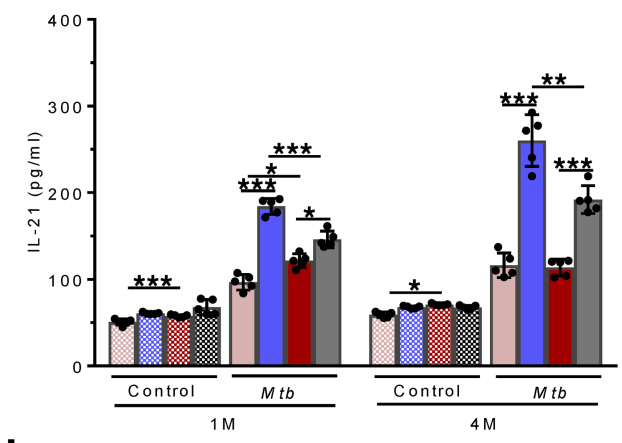

I

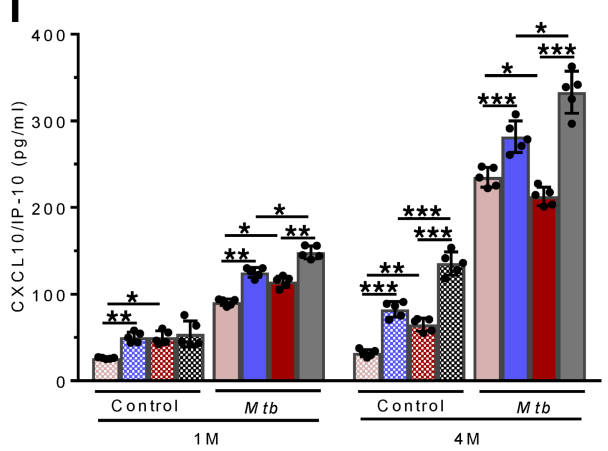

Figure 3. BCG vaccination alters cytokine and chemokine levels in T2DM mice infected with $\boldsymbol{M t b}$. PBS control, BCG-vaccinated, T2DM, or BCG-vaccinated T2DM mice were infected with 100 CFU of aerosolized Mtb H37Rv. (A) At 1 and 4 months p.i., lung homogenates from PBS control, BCG-vaccinated control, T2DM, BCG-vaccinated T2DM, infected control, infected T2DM, infected BCG-vaccinated control, and infected BCG-vaccinated T2DM mice were collected, and cytokine and chemokine levels were measured in a multiplex ELISA.

(A-I) Quantitative results of the cytokines and chemokines IL-1 $\beta$ (A), IFN- $\gamma$ (B), IL-17 (C), IL-6 (D), IL-13 (E), IL-15 (F), IL-21 (C), IL-22 (H), and CXCL10/IP-10 (I) are shown. Experiments were performed 2 times, and each time, 2-3 mice per group were used. The data are shown as mean \pm SDs of $n=5$ mice per group. The statistical analysis was performed by 2-way ANOVA, followed by Tukey's multiple comparisons test. ${ }^{*} P<0.05$, ${ }^{* *} P<0.01$, and ${ }^{* *} P<0.001$ 

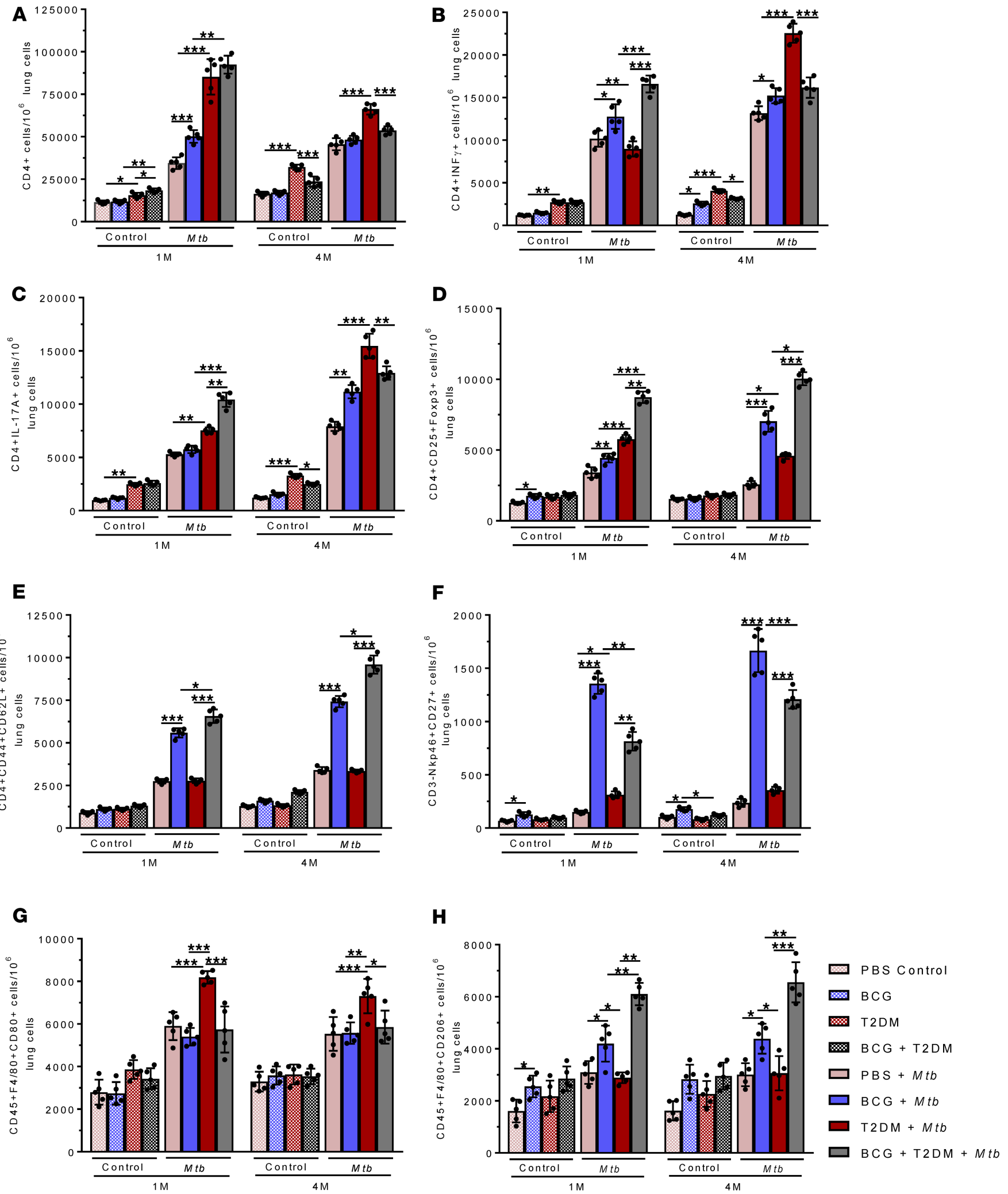

Figure 4. BCC vaccination induces increased infiltration of Tregs and $M 2$ macrophages in the lungs of $\mathbf{M t b}$-infected T2DM mice. PBS control, BCG-vaccinated, T2DM, or BCG-vaccinated T2DM mice were infected with 100 CFU of aerosolized Mtb H37Rv. (A) At one and 4 months p.i., the lungs from PBS control, BCG-vaccinated control, T2DM, BCG-vaccinated T2DM, infected control, infected T2DM, infected BCG-vaccinated control, and infected BCG-vaccinated T2DM mice were isolated. (A-H) The absolute numbers per $1 \times 10^{6}$ total lung cells of the following leukocyte populations were determined by flow cytometry: CD4+ (A), CD4+IFN- $\gamma^{+}$

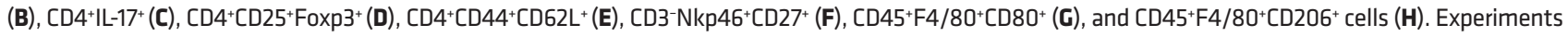
were performed 2 times, and each time, 2-3 mice per group were used. The data are shown as mean \pm SDs of $n=5$ mice per group. The statistical analysis was performed by 2-way ANOVA, followed by Tukey's multiple comparisons test. ${ }^{*} P<0.05,{ }^{* *} P<0.01$, and ${ }^{* * *} P<0.001$. 


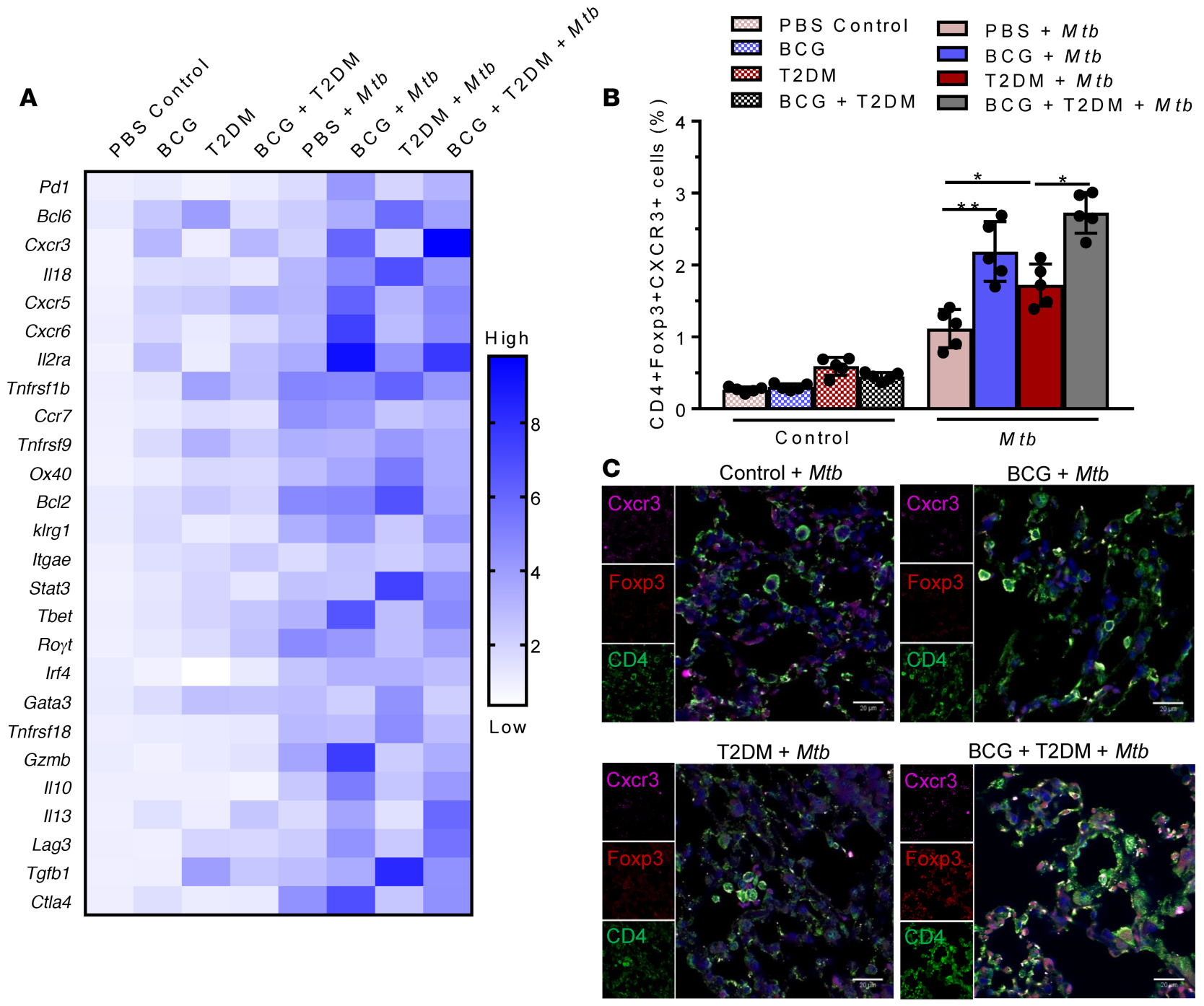

Figure 5. BCG vaccination-induced infiltration of CXCR3 ${ }^{+}$Tregs in the lungs of $\mathbf{M t b}$-infected T2DM mice. PBS control, BCG-vaccinated, T2DM, or BCG-vaccinated T2DM mice were infected with 100 CFU of aerosolized Mtb H37Rv. (A) At 4 months p.i., total RNA from the lungs of PBS control, BCG-vaccinated control, T2DM, BCG-vaccinated T2DM, infected control, infected T2DM, infected BCG-vaccinated control, and infected BCG-vaccinated T2DM mice was isolated. Heatmap data show quantitative PCR analysis (mean value [ $n=5$ mice per group] of log fold change is shown) for various molecules expressed by Tregs. (B) Tregs were isolated from the lungs of uninfected and infected mice from all the above groups of mice as described in Methods, and the percentage of $\mathrm{CXCR3^{+ }}$ Tregs was determined by flow cytometry. Experiments were performed 2 times, and each time, 2-3 mice per group were used. The data are shown as mean \pm SDs of $n=5$ mice per group. The statistical analysis was performed by 1-way ANOVA, followed by Tukey's multiple comparisons test. ${ }^{*} P<0.05$ and ${ }^{*} P<0.01$. (C) At 4 months p.i., the lungs from Mtb-infected PBS control, BCG-vaccinated control, T2DM, and BCG-vaccinated T2DM mice were isolated and formalin fixed. Paraffin-embedded tissue sections were prepared and analyzed by confocal

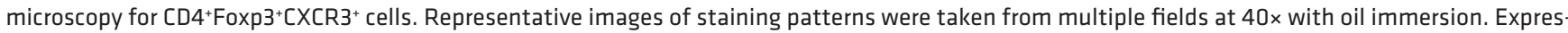
sion of CD4 (green), Foxp3 (red), and CXCR3 (magenta) are shown. Scale bar: $20 \mu \mathrm{m}$.

IL-13 receptor (IL-13R) signaling. Splenocytes from BCG-vaccinated nondiabetic mice and BCG-vaccinated T2DM mice were stimulated with or without $\gamma M t b$. Some of the $\gamma M t b$-stimulated cells were cultured with isotype-matched or anti-IL-13R mAbs. After 72 hours, M1 and M2 macrophage phenotypes were determined by flow cytometry. BCG-vaccinated T2DM macrophages expressed a significantly higher M2-like phenotype (Arg1 ${ }^{+}$cells) when compared with nondiabetic macrophages after stimulation with $\gamma M t b$ (6.04 \pm 0.94 versus $2.52 \pm 0.35 \%, P<0.001)$. In contrast, anti-IL-13R mAb treatment inhibited the M2-like macrophage phenotype expansion in BCG-vaccinated T2DM mice (Figure 7C). We further found that IL-13R is highly expressed by M2 phenotype macrophages $\left(\mathrm{CD} 45^{+} \mathrm{F} 4 / 80^{+} \mathrm{Arg} 1^{+}\right.$cells) in BCG-vaccinated T2DM mice compared with BCG-vaccinated nondiabetic mice (Figure 7D).

To confirm the above findings, we isolated macrophages ( $\mathrm{F} 4 / 80^{+}$cells) from the spleens of BCG-vaccinated nondiabetic mice and cocultured them with either autologous Tregs or Tregs from 
A

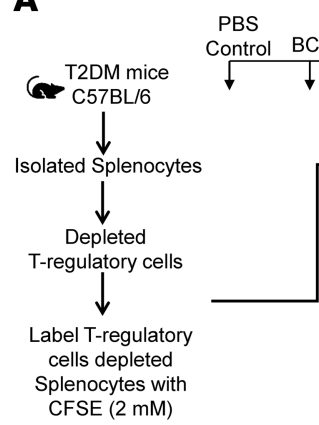

B

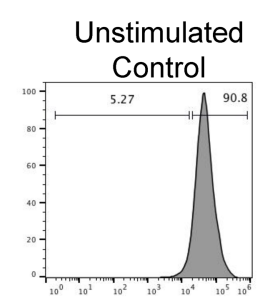

PBS Control
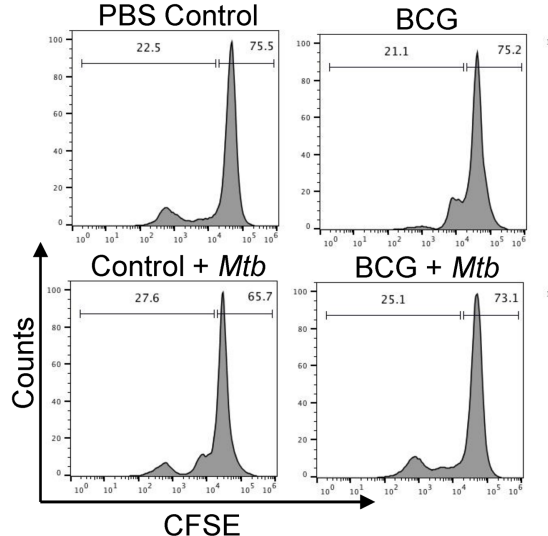

$\mathrm{BCG}+\mathrm{PBS}+\mathrm{BCG}+\mathrm{T} 2 \mathrm{DM}+\mathrm{BCG}+$

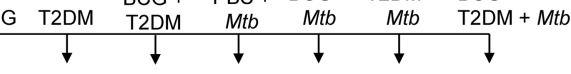

Isolated T-regulatory cells

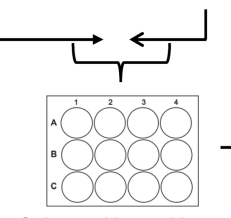

Culture with or without YMtb for $72 \mathrm{~h}$

$\longrightarrow \mathrm{CD} 4+\mathrm{T}$ cell proliferation (Flow Cytometry)

Cytokine/Chemokine analysis (Multiplex ELISA)

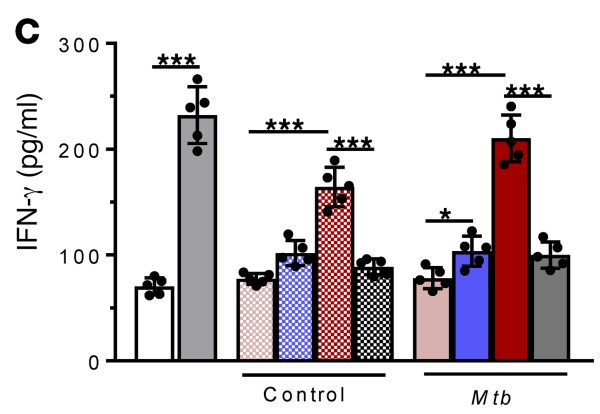

D

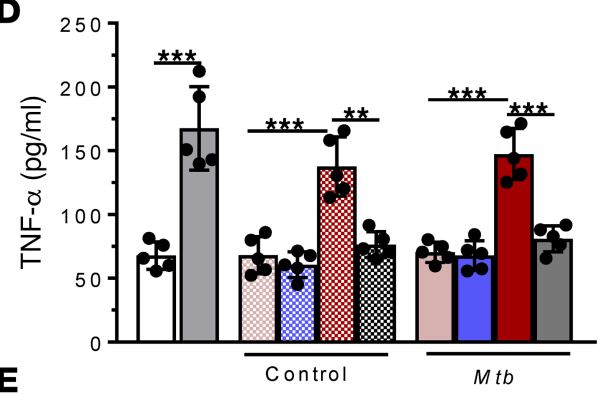

T2DM BCG+T2DM
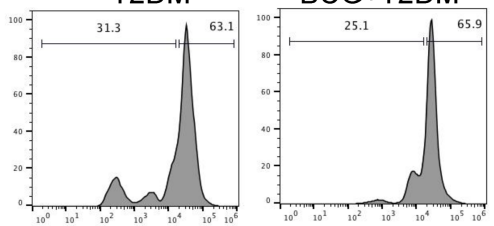

$\mathrm{T} 2 \mathrm{DM}+\mathrm{Mtb}$
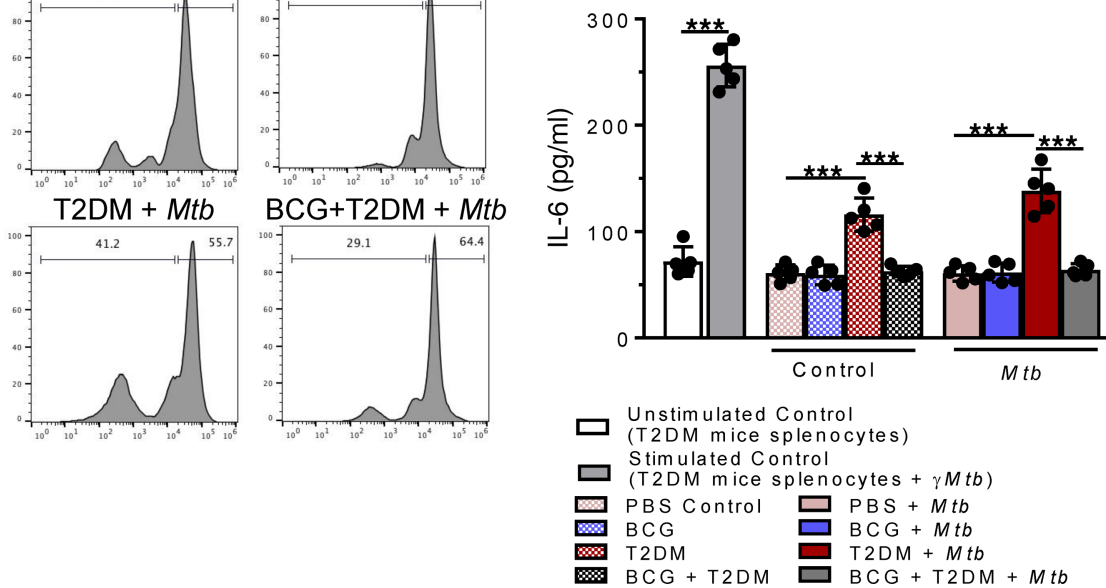

Figure 6. Tregs from T2DM mice are less immunosuppressive. PBS control, BCG-vaccinated, T2DM, or BCG-vaccinated T2DM mice were infected with 100 CFU of aerosolized Mtb H37Rv. Tregs were isolated from the lungs of PBS control, BCG-vaccinated control, T2DM, BCG-vaccinated T2DM, infected control, infected T2DM, infected BCG-vaccinated control, and infected BCG-vaccinated T2DM mice. (A and B) Treg-depleted splenocytes were isolated from T2DM mice and labeled with CFSE ( $2 \mathrm{mM}$ ) and cultured with $\gamma$-irradiated $\mathrm{Mtb}$ H37Rv (10 $\mu \mathrm{g} / \mathrm{mL})$ in the presence of Tregs from all the above groups of mice at a 1:1 ratio. After 72 hours, supernatants were collected, and flow cytometry was performed on cells to determine the CFSE+CD4+ ${ }^{+}$cell proliferation. Histogram plots showing the $T$ cell proliferation and bar graph depicting percentage of CD4+ $4^{+}$cell proliferation. (C-E) IFN- $\gamma$, TNF- $\alpha$, and IL- 6 levels in culture supernatants were determined by multiplex ELISA. Experiments were performed 2 times, and each time, $2-3$ mice per group were used. The data are shown as mean \pm SDs of $n=5$ mice per group. The statistical analysis was performed by 1-way ANOVA, followed by Tukey's multiple comparisons test. ${ }^{*} P<0.05$, ${ }^{* *} P<0.01$, and ${ }^{* * *} P<0.001$.

BCG-vaccinated T2DM mice in the presence of $\gamma M t b$. Tregs from BCG-vaccinated T2DM mice significantly expanded macrophages to the $\mathrm{M} 2$ phenotype $\left(\mathrm{CD} 45^{+} \mathrm{F} 4 / 80^{+} \mathrm{Arg} 1^{+}\right.$cells $)$when compared with autologous Tregs $(P<0.01)$ (Figure $7 \mathrm{E}$ and Supplemental Figure 14, A and B).

Furthermore, we determined whether the above expansion depends on IL-13R signaling. Macrophages from BCG-vaccinated T2DM mice were isolated and cultured with $\gamma M t b$ in the presence or absence of autologous Tregs and with isotype control or anti-IL-13R antibodies. After 72 hours, the M1 and M2 phenotypes were measured by flow cytometry, and proinflammatory cytokine levels were measured in culture supernatants. As shown in Figure 7F and Supplemental Figure 14C, in the presence of Tregs and $\gamma M t b$, the anti-IL-13R mAb significantly reduced the expansion of the M2 phenotype and increased TNF- $\alpha$ and IL- 6 production.

Finally, we cultured macrophages from BCG-vaccinated T2DM mice with $\gamma M t b$ in the presence or absence of recombinant IL-13 (rIL-13). As shown in Figure 7G and Supplemental Figure 14D, in the 
A
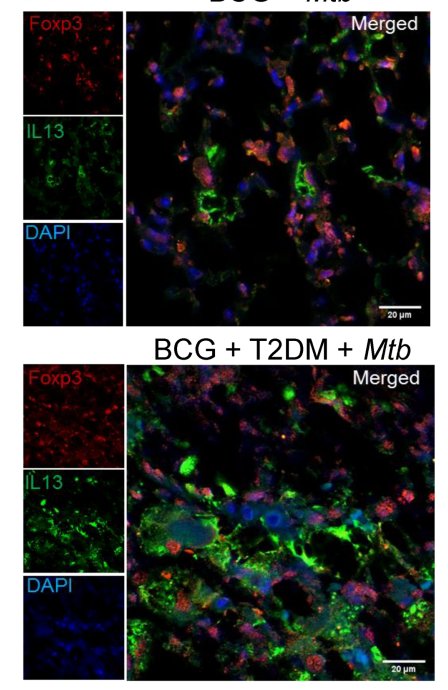

E

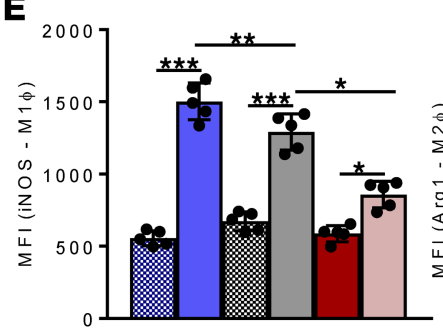

$\mathbf{F}$

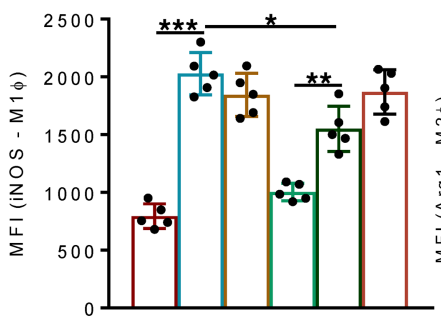

B
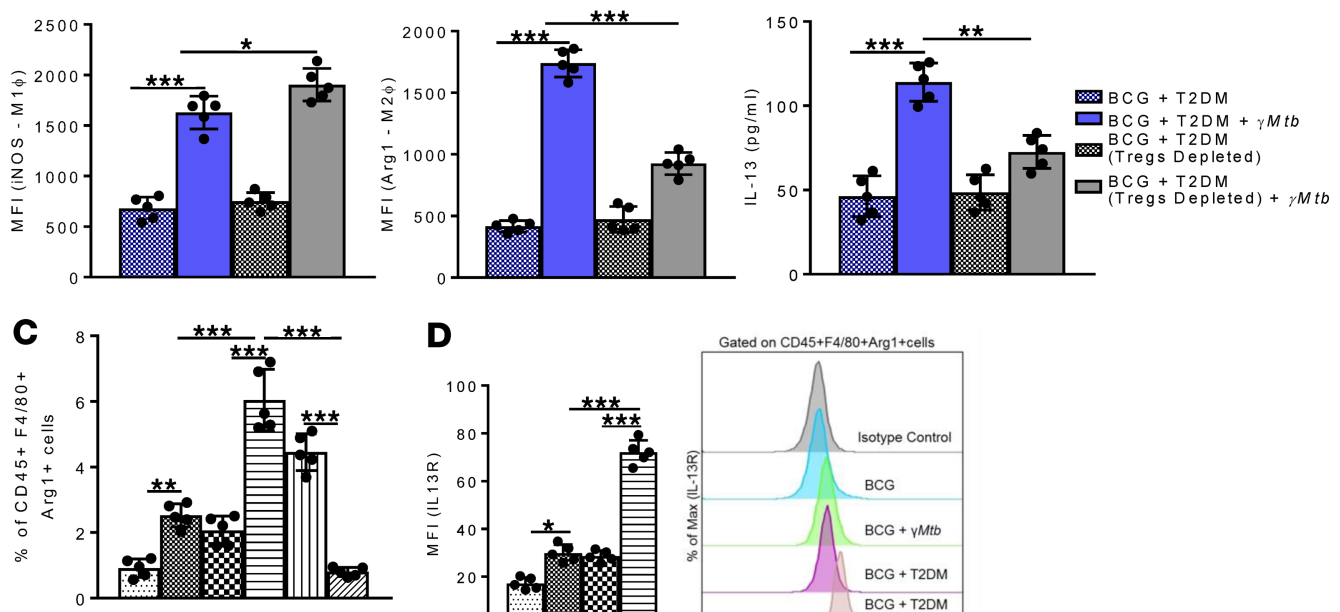

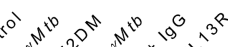

$8^{\circ} 8^{\circ} 0^{\circ}$

D
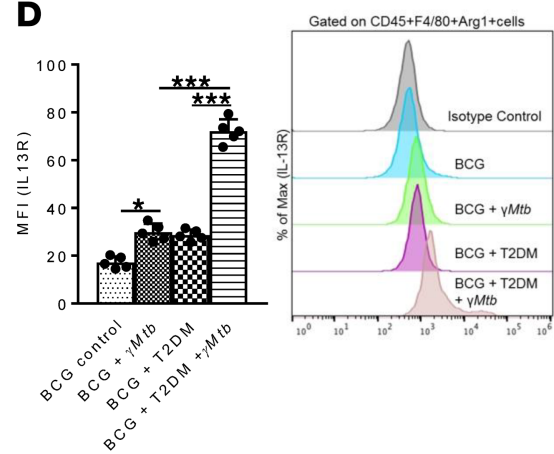

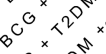

$8^{0} \times{ }^{0} \times 2^{\circ}$

$8^{0^{\circ}}$

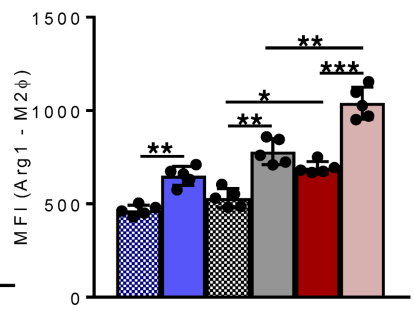

$m \phi(B C G)$

$\square M \phi(B C G)+\gamma M t b$

$M \phi(B C G)+\operatorname{Tregs}(B C G)$

$\square M(B C G)+$ Tregs $(B C G)+\gamma M t$

$M \phi(B C G)+\operatorname{Tregs}(B C G+T 2 D M)$

$\square M \phi(B C G)+\operatorname{Tregs}(B C G+T 2 D M)+\gamma M t b$

G

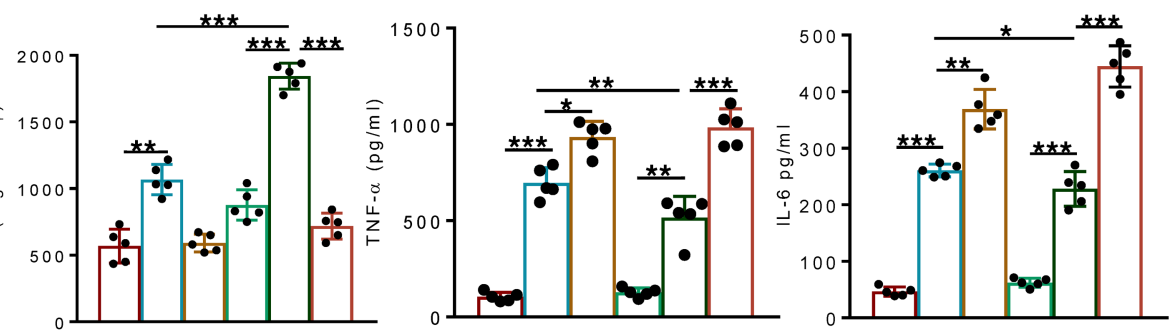

$\square M \phi(B C G+T 2 D M)$

$\square M \phi(B C G+T 2 D M)+I g G+\gamma M t b$

$\square M \phi(B C G+T 2 D M)+$ Tregs

$M \phi(B C G+T 2 D M)+a n t i-I L-13 R+\gamma M t b$

$\square M \phi(B C G+T 2 D M)+T r e g s+I g G+\gamma M t b$

$M \phi(B C G+T 2 D M)+$ Tregs + anti-IL-13R + $M M t b$

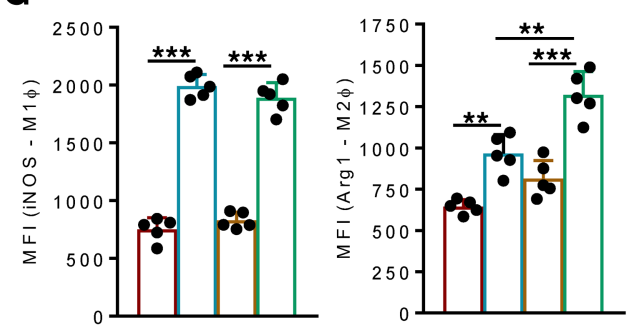

$\square M \phi(B C G+T 2 D M)$

$\square M_{\phi}(B C G+T 2 D M)+\gamma M t b$

$M \phi(B C G+T 2 D M)+r l L-13$
$M \phi(B C G+T 2 D M)+r l L-13+\gamma M t b$

Figure 7. IL-13 produced by Tregs is involved in the expansion of antiinflammatory M2 macrophages. (A) At four months p.i., the formalin fixed and paraffin embedded lungs sections from Mtb-infected BCG-vaccinated and BCG-vaccinated T2DM were analyzed by confocal microscopy for Foxp3+IL13+ cells. Foxp3 (red), IL-13 (green), and DAPI (blue) expression is shown. Scale bar: $20 \mu \mathrm{m}$. BCG-vaccinated T2DM mouse splenocytes were isolated and cultured at $1 \times 10^{6}$ cells/well in RPMI-1640 containing penicillin (Invitrogen) and $10 \%$ heat-inactivated FCS, with or without $\gamma$-irradiated Mtb H37Rv $(10 \mu \mathrm{g} / \mathrm{mL})$ at $37^{\circ} \mathrm{C}$ 
and $5 \% \mathrm{CO}_{2}$. In some wells, Treg-depleted splenocytes were stimulated with $\gamma$-irradiated $\mathrm{Mtb}(10 \mu \mathrm{g} / \mathrm{mL})$. After 72 hours, the cells were collected and phenotypically characterized for M1/M2 macrophages, and the supernatants were used to measure levels of the cytokine IL-13. (B) Mean fluorescence intensity (MFI) values showing the $\mathrm{CD} 45^{+} \mathrm{F} 4 / 80^{+} \mathrm{NNos}^{+}$and $\mathrm{CD} 45^{+} \mathrm{F} 4 / 80^{+} \mathrm{Arg} 1^{+}$cells, and IL-13 levels in culture supernatants, were measured by ELISA. (C) BCG-vaccinated nondiabetic and BCG-vaccinated T2DM mouse splenocytes were stimulated with $\gamma$-irradiated Mtb H37Rv $(10 \mu \mathrm{g} / \mathrm{mL})$ and treated with anti-IL-13R (10 $\mu \mathrm{g} / \mathrm{mL}$ ) for 72 hours. After 72 hours, flow cytometry was performed to determine the M2 macrophage phenotype (CD45 ${ }^{+} \mathrm{F} 4 / 80^{+} \mathrm{Arg} 1^{+}$cells). (D) MFI values and histogram plot showing IL-13R expression gated on $\mathrm{CD} 45^{+} \mathrm{F} 4 / 80^{+} \mathrm{Arg} 1^{+}$cells. (E) Macrophages isolated from BCG-vaccinated mice were cocultured with autologous Tregs or Tregs isolated from BCG-vaccinated T2DM mice and stimulated with $\gamma$-irradiated Mtb $(10 \mu \mathrm{g} / \mathrm{mL})$. After 72 -hour incubation, the macrophages were analyzed for M1 and M2 phenotype by flow cytometry. MFI values showing the CD45+ $\mathrm{F} 4 / 80^{+} \mathrm{iNos}{ }^{+}$cells and $\mathrm{CD} 45^{+} \mathrm{F} 4 / 80^{+} \mathrm{Arg} 1^{+}$cells. (F) For neutralization studies, BCG-vaccinated T2DM mouse macrophages were stimulated with $\gamma$-irradiated Mtb (10 $\mu \mathrm{g} / \mathrm{mL})$ with or without autologous Tregs, and in some wells, IgG control or anti-IL-13R $(10 \mu \mathrm{g} / \mathrm{mL})$ antibodies were added. After 72 hours of incubation, the M1 and M2 phenotypes of cultured macrophages were determined by flow cytometry, and TNF- $\alpha$ and IL- 6 levels were measured by ELISA in the culture supernatants. rlL-13 (10 ng/mL) was added to the macrophages from BCG-vaccinated T2DM mice and stimulated with $\gamma$-irradiated Mtb $(10 \mu \mathrm{g} / \mathrm{mL})$. (G) After 72 hours of incubation, the M1 and M2 phenotypes of cultured macrophages were determined by flow cytometry, and MFI values for CD45+F4/80+iNos ${ }^{+}$cells and $\mathrm{CD}^{+} 45^{+} \mathrm{F} 4 / 80^{+} \mathrm{Arg} 1^{+}$cells are shown. Experiments were performed 2 times, and each time, 2-3 mice per group were used. The data are shown as mean \pm SDs of $n=5$ mice per group. The statistical analysis was performed by 1-way ANOVA, followed by Tukey's multiple comparisons test. ${ }^{*} P<0.05,{ }^{* *} P<0.01$, and ${ }^{* * *} P<0.001$.

presence of rIL-13 and $\gamma M t b$, macrophages significantly expanded to the M2 phenotype. These results suggest that Tregs from BCG-vaccinated T2DM mice induce the antiinflammatory macrophage phenotype and reduce excessive inflammation.

\section{Discussion}

In the current study, we investigated whether BCG vaccination has any effect on immune responses to $M t b$ infection in T2DM mice. We found that BCG vaccination enhanced the survival of Mtb-infected T2DM mice compared with that of unvaccinated $M t b$-infected T2DM mice. The enhanced survival of BCG-vaccinated Mtb-infected T2DM mice was due to reduced inflammation. BCG vaccination significantly expanded the IL-13-producing CXCR $3^{+}$Tregs in the lungs of Mtb-infected T2DM mice. Furthermore, we found that rIL-13 converted proinflammatory M1 macrophages to antiinflammatory M2 macrophage phenotype. In contrast, anti-IL-13R antibody inhibited this conversion and enhanced the IL- 6 and TNF- $\alpha$ production. For the first time to our knowledge, our current findings demonstrate that BCG vaccination can prevent excess inflammation and early death of T2DM mice infected with $M t b$.

T2DM alters the innate and adaptive immune responses against TB and makes the host more susceptible to developing active TB disease and less responsive to antimycobacterial treatments $(8,12,15,34-37)$. TB patients with T2DM have hyperinflammatory responses $(11,15)$. Previously, we developed an experimentally induced nonobese T2DM model in WT C57BL/6 mice and found that Mtb infection in T2DM mice drives pathological immune responses and mortality (8). We also demonstrate that our findings with this current mouse model are applicable to human $\operatorname{T} 2 \mathrm{DM}(8,16)$. However, no information is available about the immune mechanisms that regulate inflammation in T2DM hosts infected with Mtb.

BCG is given to all children born in countries where TB is endemic, as it protects against the most severe manifestations of disease. The efficacy of BCG vaccination varies from $0 \%-80 \%(21,38-41)$, and the reasons for this variability remain unclear (38-40). Neonatal BCG vaccination induces a complex pattern of cytokine expression by T cells $(41,42)$. One study showed that delaying BCG vaccination from birth to 10 weeks of age enhances the quantitative and qualitative BCG-specific T cell response (43). Another study found that BCG vaccination at birth induced a broad range of Th1/Th2/Th17/Tregs against mycobacteria, but the Th1/Th17 response was reduced when vaccine administration was delayed (44). BCG vaccination in infants induces IL-4, IL-5, and IL-13 production (42, 44-46). Infants have significantly higher numbers of Tregs, and CD4 ${ }^{+}$Tregs are induced upon BCG vaccination in newborns and adults $(19,47-49)$. The current findings further demonstrate that BCG vaccination reduces the inflammation and mortality of $M t b$-infected T2DM mice. The reduced inflammation and mortality is associated with the expansion of a subset of IL-13-producing CXCR3 ${ }^{+}$Tregs.

Tregs are crucial for the maintenance of peripheral tolerance and homeostasis $(50,51)$. During $M t b$ infection, Tregs inhibit effective immune responses $(19,52,53)$. Tregs express various homing chemokine receptors such as CCR4, CCR8, and CXCR3 and are recruited to the site of infection to downregulate Th1 cell function to counterbalance undesirable tissue destruction and prevent immunopathology (54-57). Transcription factor T-bet-deficient mice do not have CXCR3 ${ }^{+}$Tregs, and the IFN- $\gamma$ receptor (IFN- $\gamma \mathrm{R}$ ) plays an important role in T-bet expression in Tregs (55). IFN- $\gamma$ induces the production of the CXCR3 ligand CXCL10 to recruit $\mathrm{CXCR} 3^{+}$cells to the site of inflammation. In our study, we found 
that BCG-vaccinated $M t b$-infected T2DM mouse lungs had significantly higher CXCL10 (Figure 3I) and $\mathrm{CXCR}^{+}$Tregs compared with those of all other groups of control and $M t b$-infected mice. Our results suggest that excessive inflammatory conditions in $M t b$-infected T2DM mice enhance the expansion of $\mathrm{CXCR}^{+}$Tregs that are recruited to the lungs to inhibit inflammation and enhance the survival of BCG-vaccinated mice. A similar type of amelioration of graft versus host disease (GVHD) was seen when CXCR $3^{+}$Tregs were adoptively transferred to mice (54).

The inflammatory environment can hamper the suppressive ability of Tregs (58). Accordingly, we found that the immunosuppressive capacity of Tregs of $M t b$-infected T2DM mice was significantly less compared with $M t b$-infected non-T2DM mice. In contrast, prior BCG vaccination restored the immunosuppressive capacity of T2DM mice infected with $M t b$ similar to $M t b$-infected non-T2DM mice (Figure 6B). Prior BCG-vaccinated $M t b$-infected T2DM and non-T2DM mice Tregs expressed similar levels of IL-10. In contrast, BCG-vaccinated T2DM mice infected with Mtb expressed significantly higher amounts of IL-13 protein and mRNA compared with all other groups of mice (Figure 5A). Our current findings demonstrate that prior BCG vaccination inhibits excess inflammatory responses and preserves the immunosuppressive function of Tregs of $M t b$-infected T2DM mice. In addition to restoring the immunosuppressive capacity of Tregs, BCG vaccination significantly enhanced the capacity of Tregs to produce IL-13 in T2DM mice infected with $M t b$ compared with that of all other groups of control and Mtb-infected mice (Figure 5A and Figure 7B).

IL-13 is a $13-\mathrm{kDa}$ protein with structural homology with IL-4. Various cell populations, such as CD4, NK, and mast cells, produce IL-13 (59). In the current study, we found that Tregs are the major source of IL-13 in BCG-vaccinated T2DM mice infected with Mtb. A previous report indicated that IL-13 skews macrophage polarization toward the M2 phenotype $(60,61)$. We also found that rIL-13 can convert Mtb-cultured macrophages from BCG-vaccinated T2DM mice to the M2 phenotype (Figure 7G and Supplemental Figure 14C). Lung macrophages from BCG-vaccinated T2DM mice expressed higher levels of IL-13R, which could indicate a propensity of response toward IL-13 (Figure 7C). Furthermore, anti-IL-13R antibody inhibited the conversion of macrophages from the BCG-vaccinated T2DM mice from M1 to the M2 phenotype, and rIL-13 expanded M2 macrophage phenotype. The IL-13R $\alpha 1$ chain is functionally essential for the induction of alternatively activated macrophages (M2) through the IL-13 pathway in peritonitis and lung injury $(62,63)$. Treg-mediated production of IL-13 is required to prevent mortality after acute lung injury by controlling local levels of G-CSF, IL-6, and MCP-1 and inhibiting accumulation of Ly6C ${ }^{\text {hi }}$ monocytes (64). Our current study further demonstrates that BCG vaccination and Mtb infection in T2DM mice induce Tregs to produce IL-13. IL-13R signaling is associated with the expansion of the M2 macrophage phenotype to inhibit excess inflammation in BCG-vaccinated T2DM mice. These findings suggest a role for IL-13R signaling during $M t b$ infection in vaccinated T2DM mice. Signaling pathways like JAK-STAT pathway classically activate M2 macrophage polarization through IL-4 and IL-13R signaling and upregulate the M2 macrophage genes, including Ym1, Arg1, Fizz1, IL-10, and MGL1 (65). Further studies are needed to determine the exact in vivo role of IL-13R signaling pathway in the induction of the M2 macrophage phenotype. It also remains to be determined the mechanisms of BCG-induced IL-13 secretion by Tregs.

M1/M2 macrophage balance modulates cancer and metabolic, infectious, and inflammatory diseases (6668). M1 macrophages have potent cytotoxic functions and mediate resistance against infection (69-71). In contrast, alternatively activated M2 phenotype polarization resolves hyperinflammation (72-74). Mtb infection suppresses macrophages to avoid cytotoxic functions and evade the cellular immune response or repolarize M1 macrophages toward an M2 phenotype (69, 75-78). T2DM-associated inflammation is characterized by an increased number of M1 macrophages $(79,80)$. In the current study, we found that uncontrolled infiltration of M1 macrophages in the lungs of T2DM mice after Mtb infection was associated with lung pathology. However, vaccination of T2DM mice with BCG expanded M2 macrophages in an IL-13-dependent manner (Figure 7). Our current study suggests a role for IL-13/IL-13R signaling in reducing excess inflammation and mortality of $M t b$-infected T2DM hosts through the expansion of the M2 macrophage phenotype.

In conclusion, using the BCG-vaccinated T2DM mouse model, we found that BCG vaccination of $M t b$-infected T2DM mice enhanced survival and reduced inflammation. The enhanced survival of BCG-vaccinated T2DM mice infected with $M t b$ was associated with the expansion of a subset of CXCR3-expressing and IL-13-producing Tregs. Further understanding of this BCG-mediated immunosuppressive mechanisms will help to develop an adequate prophylactic or therapeutic agent to prevent inflammation and mortality of the T2DM host infected with Mtb. 


\section{Methods}

Animals. Specific pathogen-free 4- to 6-week-old female C57BL/6 mice were purchased from the Jackson Laboratory and housed at the animal facility at the University Texas Health Science Center at Tyler. Sample sizes were chosen based on previous published studies $(8,16)$. For survival studies, 15 mice/group were used, while for immunophenotyping and histological analysis, 5 mice/group were used. All mice were maintained on a standard rodent chow diet (LabDiet, catalog 5053, 4.07 kcal/gm) during the entire experiment. After $M t b$ infection, all mice were housed randomly at 5 animals per cage in high-efficiency particulate air (HEPA) filtered racks in certified animal biosafety level 3 (ABSL-3) laboratories. The weights were measured at 15-day intervals, and blood glucose levels of the mice were recorded monthly after induction of T2DM.

Antibodies and other reagents. For flow cytometry, we used anti-CD3 (catalog 100306), anti-CD8 (catalog 100712), anti-CD4 (catalog 100422), anti-IL-4 (catalog 504103), anti-IL-17A (catalog 506927), anti-CD44 (catalog 103006), anti-CD62L (catalog 104406), anti-CD25 (catalog 102036), anti-Foxp3 (catalog 320014), anti-B220 (catalog 103206), anti-IgM (catalog 406509), anti-Nkp46 (catalog 137618), anti-CD27 (catalog 124209), anti-NK1.1 (catalog 108728), anti-CD11b (catalog 101226), anti-Ly6G (catalog 108443), antiCD11c (catalog 117334), anti-F4/80 (catalog 123132), anti-CXCR3 (catalog 126521), anti-CD45 (catalog 103116), anti-CD80 (catalog 104733), and anti-CD206 (catalog 141703) antibodies (all from BioLegend). Anti-Arg1 (catalog 56-369-782) and anti-iNOS (catalog 50-112-2349) were obtained from Thermo Fisher Scientific. Anti-IFN- $\gamma$ (catalog 17-7311-82) and anti-IL-21 (catalog 12-7211-80) were obtained from eBioscience. For confocal microscopy, anti-CD4 (Novus, catalog NBP225191SS), anti-Foxp3 (Novus, catalog NB10039002T), anti-CXCR3 (Novus, catalog NBP2-12239), anti-iNOS (Santa Cruz Biotechnology Inc., catalog sc-7271), anti-Arg1 (Santa Cruz Biotechnology Inc., catalog sc-271430), anti-F4/80 (Cell Signaling Technology, catalog 71299), anti--IL-13 (Abcam, catalog ab106732), and anti-IL-13R (R\&D Systems; catalog AF539) and secondary antibodies (goat anti-rat IgG [H+L], Alexa 647 [catalog A21247]; goat anti-rabbit IgG [H+L], Alexa Fluor 488 [catalog A11008]; and goat anti-mouse IgG [H+L], Alexa Fluor 594 [catalog A11032]) were obtained from Invitrogen, and Fluoroshield mounting medium with DAPI (Abcam, catalog ab104139) was used for the staining nuclei. For neutralization studies, an anti-IL-13 antibody was obtained from Thermo Fisher Scientific (catalog 16-7135-81). rIL-13 (carrier free) was obtained from BioLegend (catalog 575902). $\gamma M t b$ was obtained from BEI resources for in vitro stimulation assays. Streptozotocin (STZ) and nicotinamide (NA) were obtained from MilliporeSigma.

$B C G$ vaccination and aerosol infection with $\mathrm{Mtb} H 37 R v$. Six- to 8-week-old C57BL/6 mice were immunized s.c. with $1 \times 10^{6} \mathrm{CFU}$ of BCG in $100 \mu \mathrm{L}$ PBS or with PBS alone. Three months of after vaccination, some of the mice were induced with T2DM. For infection studies, some of the BCG-vaccinated T2DM mice were infected with $M t b \mathrm{H} 37 \mathrm{Rv}$ using an aerosol exposure chamber as described previously $(81,82)$. Briefly, Mtb H37Rv was grown to the mid-log phase in liquid culture medium and then frozen in aliquots at $-70^{\circ} \mathrm{C}$. Bacterial counts were determined by plating on $7 \mathrm{H} 10$ agar supplemented with oleic albumin dextrose catalase (OADC). For infection, the bacterial stocks were diluted in $10 \mathrm{~mL}$ of normal saline $\left(0.5 \times 10^{6}\right.$ $\mathrm{CFU} / \mathrm{mL}, 1 \times 10^{6} \mathrm{CFU} / \mathrm{mL}, 2 \times 10^{6} \mathrm{CFU} / \mathrm{mL}$, and $4 \times 10^{6} \mathrm{CFU} / \mathrm{mL}$ ) and placed in a nebulizer within an aerosol exposure chamber custom made by the University of Wisconsin (Madison, Wisconsin, USA). In the preliminary studies, groups of 3 mice were exposed to the aerosol at each concentration for 15 minutes. After 24 hours, the mice were euthanized, and homogenized lung samples were plated on 7H10 agar plates supplemented with OADC. CFUs were counted after 21 days of incubation at $37^{\circ} \mathrm{C}$. The aerosol concentration that resulted in $\sim 100$ bacteria in the lungs was used for further studies.

Induction of T2DM. T2DM was induced by combined administration of STZ and NA as described previously (8). STZ was dissolved in $50 \mathrm{mM}$ citric acid buffer and administered (180 $\mathrm{mg} / \mathrm{kg}$ of body weight) i.p. 3 times, with an interval of 10 days between doses. NA was dissolved in saline and administered i.p. (60 mg/kg of body weight) 15 minutes before the STZ injections. Blood glucose levels were monitored using a glucometer at monthly intervals for up to 10 months. Mice were considered as T2DM if their blood glucose level was $\geq 250 \mathrm{mg} / \mathrm{dL}$, and control mouse blood glucose levels were regularly measured between 80 and $125 \mathrm{mg} / \mathrm{dL}$.

Measurement of serum insulin and lipid profiles. Serum insulin levels in fasting (6 hour) mice were measured using a Mercodia ultrasensitive insulin ELISA kit (Mercodia AB Uppsala). Serum free fatty acids, cholesterol, and triglycerides were measured using either a fluorometric or colorimetric assay according to the manufacturer's instructions (Cayman Chemicals). 
Preparation of lung and spleen cells. Lungs and spleens were harvested from the PBS control, BCG-vaccinated, T2DM, and BCG-vaccinated T2DM mice that were uninfected and Mtb H37Rv infected at the indicated time points and were placed into $60-\mathrm{mm}$ dishes containing $2 \mathrm{~mL}$ of HBSS (Thermo Fisher Scientific). The tissues were minced with scissors into pieces no larger than $2-3 \mathrm{~mm}$, and the fluid was discharged onto a 70- $\mu \mathrm{m}$ filter (BD Biosciences) that had been prewetted with $1 \mathrm{~mL}$ of PBS containing 0.5\% BSA (MilliporeSigma), suspended over a 50-mL conical tube (Thermo Fisher Scientific, 06-44318). The syringe plunger was then used to gently disrupt the lung and spleen tissues before washing the filter with $2 \mathrm{~mL}$ of cold PBS $/ 0.5 \%$ BSA. The total number of viable cells in the lungs was determined with the trypan blue exclusion method. For flow cytometry experiments, we gated on the total leukocytes and measured various cell populations (83).

Multiplex ELISA to measure cytokines and chemokines. In the lung homogenates, 36 cytokines and chemokines were measured using a multiplex ELISA kit (EPX360-26092-901, ProcartaPlex, Thermo Fisher Scientific). The cytokines and chemokines analyzed by multiplex ELISA were IFN- $\gamma$, IL-1 $\alpha$, IL-1 $\beta$, IL-3, IL-2, IL-4, IL-5, IL-6, IL-9, IL-10, IL-12 [p70], IL-13, IL-15, IL-17, IL-18, TNF- $\alpha$, IFN- $\alpha$, IL-22, IL-23, IL-27, IL-28, IL-31, GM-CSF, ENA-78, G-CSF, M-CSF, LIF, Eotaxin, Gro- $\alpha$, IP-10, MCP-1, MCP-3, MIP-1 $\alpha$, MIP-1 $\beta$, MIP-2, and RANTES, and the IFN- $\beta$, IL-7, IL-13, and TGF- $\beta$ (Thermo Fisher Scientific) were analyzed using simplex ELISA kits.

Flow cytometry and intracellular staining. For surface staining, $1 \times 10^{6}$ cells were resuspended in $100 \mu \mathrm{L}$ of staining buffer (PBS containing 2\% heat-inactivated FBS) and antibodies. The cells were then incubated at $4^{\circ} \mathrm{C}$ for 30 minutes with appropriate surface staining markers, washed twice, and fixed in $1 \%$ paraformaldehyde (MilliporeSigma) before acquisition using an Attune NxT acoustic flow cytometer (Invitrogen). In some experiments, cells were permeabilized with $0.1 \%$ saponin (MilliporeSigma) and intracellularly stained for IFN- $\gamma$, IL-17A, IL-4, Foxp3, IL-21, IL-10, TGF- $\beta$, iNOS, and Arg1. The cells were washed and resuspended in FACS buffer and fixed in 1\% paraformaldehyde before acquisition using an Attune NxT acoustic flow cytometer (Invitrogen).

Isolation of mouse lung and spleen Tregs. Single-cell suspensions of mouse lungs and spleen cells were prepared from all the groups of mice. $\mathrm{CD} 4^{+} \mathrm{CD} 25^{+}$Tregs were isolated by magnetic beads according to the manufacturer's instructions (Miltenyi Biotec) and as described previously (84). Isolated cells contained $>97 \% \mathrm{CD}^{+} \mathrm{CD}^{2} 5^{+} \mathrm{Foxp}^{+}$cells, as measured by flow cytometry. The isolated Tregs were either stored in Trizol for RNA isolation or used for in vitro-stimulation studies. Based on the above method, in some experiments, we depleted the $\mathrm{CD} 4{ }^{+} \mathrm{CD} 25^{+}$cells from $M t b$-infected T2DM mouse splenocytes to determine the $\mathrm{T}$ cell proliferation efficiency.

CFSE labeling and in vitro stimulation. Treg-depleted mouse splenocytes were resuspended $\left(1 \times 10^{5}\right.$ cells $/$ $\mathrm{mL}$ ) in $0.1 \%$ BSA in PBS. CFSE (Invitrogen) was added to a final concentration of $2 \mathrm{mM}$, and cells were incubated at $37^{\circ} \mathrm{C}$ for 15 minutes. Cells were washed once with $10 \%$ FCS (MilliporeSigma) in PBS and twice with $0.1 \%$ BSA in PBS, and they were resuspended in RPMI 1640 for subsequent stimulation. Isolated Tregs from all groups of mice were cultured at a 1:1 ratio with CFSE-labeled splenocytes and stimulated with $\gamma$-irradiated $M t b(10 \mu \mathrm{g} / \mathrm{mL})$. After 72 hours of incubation, we measured $\mathrm{CFSE}^{+} \mathrm{CD} 4^{+} \mathrm{T}$ cell proliferation by flow cytometry. Culture supernatants were collected to determine the levels of cytokines and chemokines using a multiplex ELISA kit (EPX360-26092-901, ProcartaPlex, Thermo Fisher Scientific).

Detection of ESAT-6 ${ }_{4-17 a}$-specific T cells and Tregs. PE-labeled MHC II tetramers (I-A ${ }^{\mathrm{b}}$ ) containing the stimulatory residues 4-17 (QQWNFAGIEAAASA) of the early secreted antigenic target $6 \mathrm{kDa}$ (ESAT-6) of $M t b$ were obtained from the NIH Tetramer Core Facility (Emory University Vaccine Center). For Ag-specific responses and intracellular cytokine staining, cells were incubated with ESAT- $6_{4-17 a a}$ MHC II multimers (1:50 dilution) at $37^{\circ} \mathrm{C}$ in $5 \% \mathrm{CO}_{2}$ for 60 minutes in media, washed 3 times, and cultured with ESAT- $6_{4-17 a a}$ peptide in the presence of monensin. After 5 hours, cells were surface-stained for CD3, CD4, CD8, and CD25 at room temperature for 15 minutes in PBS with $0.5 \%$ BSA and $20 \%$ mouse serum, washed, and then fixed with $2 \%$ paraformaldehyde (Sigma-Aldrich) for 1 hour. After fixation, cells were permeabilized and then washed in PBS with $0.5 \%$ BSA and $0.2 \%$ saponin (MilliporeSigma). Cells were intracellularly stained with anti-IFN- $\gamma$, anti-IL-17A, and anti-Foxp3 Abs by incubating in staining medium for 15 minutes at room temperature. Cells were washed and resuspended in 1\% paraformaldehyde. Acquisition was performed in an Attune NxT acoustic flow cytometer (Invitrogen) and analyzed using FlowJo software v8.6.3 (Tree Star Inc.).

Ex vivo splenocyte stimulation and other assays. In some experiments, BCG-vaccinated T2DM mouse splenocytes were isolated and cultured in 12 -well plates at $1 \times 10^{6}$ cells/well in RPMI 1640 containing 
penicillin (Invitrogen) and 10\% heat-inactivated FCS, with or without $\gamma$-irradiated $M t b(10 \mu \mathrm{g} / \mathrm{mL})$ at $37^{\circ} \mathrm{C}$ and $5 \% \mathrm{CO}_{2}$. In some wells, Treg-depleted splenocytes were stimulated with $\gamma$-irradiated $\mathrm{Mtb}(10$ $\mu \mathrm{g} / \mathrm{mL})$ or IgG control, or IL-13R antibodies $(10 \mu \mathrm{g} / \mathrm{mL})$ were added into the $\gamma$-irradiated Mtb-stimulated Treg containing splenocytes culture. After 72 hours, the cells were collected and phenotypically characterized for M1/M2 macrophages, and the supernatants were used to measure the levels of various cytokines by multiplex ELISA.

Macrophage and Treg coculture. In another experiment, macrophages (F4/80+ cells) were isolated from the spleens of BCG-vaccinated nondiabetic or BCG-vaccinated T2DM mice with magnetic beads according to the manufacturer's instructions (Miltenyi Biotec). In some wells, macrophages isolated from BCG-vaccinated mice were cocultured with 2:1 ratio of autologous Tregs or Tregs isolated from BCG-vaccinated T2DM mice and stimulated with $\gamma$-irradiated $M t b(10 \mu \mathrm{g} / \mathrm{mL})$. After 72-hour incubation, the macrophages were analyzed for M1 and M2 phenotype by flow cytometry. For neutralization studies, macrophages from BCG-vaccinated T2DM mice macrophages were stimulated with $\gamma$-irradiated $M t b(10 \mu \mathrm{g} / \mathrm{mL})$ with or without autologous Tregs, and — in some wells - IgG control or IL-13R $(10 \mu \mathrm{g} / \mathrm{mL})$ antibodies were added. In some experiments, $\mathrm{rIL}-13(10 \mathrm{ng} / \mathrm{mL})$ was added to the macrophages from BCG-vaccinated T2DM mice and stimulated with $\gamma$-irradiated $M t b(10 \mu \mathrm{g} / \mathrm{mL})$. After 72 hours of incubation, the M1 and M2 phenotype of cultured macrophages was determined by flow cytometry, and TNF- $\alpha$ and IL- 6 levels were measured by the ELISA in the stored culture supernatants.

Real-time PCR. Total RNA was extracted from mouse whole lung tissues or isolated Tregs $\left(\mathrm{CD} 4^{+} \mathrm{CD} 25^{+}\right)$ using TRIzol (Invitrogen) according to the manufacturer's instructions. RNA was reverse transcribed (iScript Reverse Transcription SuperMix for qPCR), and real-time PCR was performed using the iTaqTM Universal SYBR Green Supermix (Bio-Rad) according to the manufacturer's instructions. Gene expression analysis was performed in QuantStudio 7 Flex (Applied Biosystems). All gene expression levels were normalized to $\beta$-actin/GAPDH internal controls in each sample, and the fold changes were calculated using the $2^{-\triangle \Delta C T}$ method. The primers used in this study are listed in Supplemental Table 2.

Histology. At the specified time points, mice were euthanized, and the harvested lungs were inflated and fixed in 10\% neutral buffered formalin (StatLab) for 48 hours to inactivate the infectious agents. Paraffin-embedded blocks were cut into $5 \mu \mathrm{m}$-thick sections. For morphometric lesion and inflammation analyses, the lung sections were stained with $\mathrm{H} \& \mathrm{E}$ and examined in a blinded manner to assess the necrotic lesions as previously described (85). A semiquantitative analysis was performed using a score from 0 (no inflammation) to 4 (severe inflammation) for each of the following criteria: alveolar wall inflammation, alveoli destruction, leukocyte infiltration, and perivascular inflammation.

Confocal microscopy. Confocal microscopy was performed to determine the infiltrated $\mathrm{CD} 4{ }^{+} \mathrm{Foxp} 3^{+} \mathrm{CX}$ $\mathrm{CR}^{+}$cells and M1/M2 macrophages in lung sections. The lung tissues were stored in $10 \%$ neutral buffered formalin; then, the samples were paraffin embedded and cut into $5 \mu \mathrm{m}$--thick sections that were deparaffinized and rehydrated. The tissue sections were subjected to heat-induced antigen retrieval in $10 \mathrm{mM}$ sodium citrate buffer ( $\mathrm{pH}$ 6.0). Then, the lung tissue sections were incubated in $0.025 \%$ Triton X-100 in PBST for 10 minutes and washed 3 times for 5 minutes each $(3 \times 5$ minutes $)$ using PBS. Nonspecific binding was blocked with $5 \%$ goat serum or BSA in PBST for 1 hour, and the slides were washed $2 \times 5$ minutes with PBS. The slides were then incubated at $4^{\circ} \mathrm{C}$ overnight in PBST with the appropriate dilutions of the following primary antibodies: anti-CD4 (1:200), anti-Foxp3 (1:100), anti-CXCR3 (1:100), anti-F4/80 (1:200), anti-iNOS (1:100), anti-Arg1 (1: 100), anti-IL-13 (1:200) and anti-IL-13R (1:200); subsequently, the slides were washed thoroughly $3 \times 5$ minutes with PBS. Then, the tissue sections were stained with the respective secondary antibodies at 1:1000 dilutions (v/v), washed again with PBS for $3 \times 5$ minutes, and mounted with fluoroshield mounting medium with DAPI. The slides were then examined and analyzed under a laser scanning confocal microscope (Zeiss LSM 510 Meta). An IgG isotype secondary control was used for all the confocal microscopy studies, and Zen 2009 software (Carl Zeiss) was used for image acquisition. The images were processed/quantified uniformly for each experiment using ImageJ NIH software. Representative images from $n=5$ mice/group are shown.

Statistics. Data analyses were performed using GraphPad Prism 8.0 (GraphPad Software Inc.). The results are expressed as the mean \pm SDs or mean \pm SEM. For normally distributed data, comparisons between groups were performed using a paired or unpaired $t$ test (2 tailed). For multiple comparisons, 1- or 2-way ANOVA followed by Tukey's multiple comparisons test was performed. Mouse survival was compared using the Kaplan-Meier log-rank test. $P<0.05$ was considered significant. 
Study approval. All animal studies were approved by the IACUC of the University of Texas Health Science Center at Tyler (protocol no. 587). The animal procedures involving the care and use of mice were undertaken in accordance with the guidelines of the NIH/OLAW (Office of Laboratory Animal Welfare).

\section{Author contributions}

RKR and RV conceived the project, designed the experiments, analyzed and interpreted data, and wrote the manuscript. RKR, RST, DT, PP, and MKM performed the experiments. DT helped in designing the experiments. RV, SM, and BS interpreted the data and edited the manuscript. All authors edited and approved the manuscript.

\section{Acknowledgments}

We thank the NIH, CRDF Global, The Cain Foundation for Infectious Disease Research, and The Department of Pulmonary Immunology for providing financial support. This work was supported by grants from the NIH (AI123310 and AI127178 RV), CRDF global, The Cain Foundation for Infectious Disease Research, and Department of Pulmonary Immunology at The University of Texas Health Science Center at Tyler, Texas, USA.

Address correspondence to: Ramakrishna Vankayalapati, Department of Pulmonary Immunology, University of Texas Health Science Center at Tyler, 11937 US Highway 271, Tyler, Texas 75708-3154, USA. Phone: 903.877.5190; Email: krishna.vankayalapati@uthct.edu.

1. Restrepo BI, Schlesinger LS. Host-pathogen interactions in tuberculosis patients with type 2 diabetes mellitus. Tuberculosis (Edinb). 2013;93 Suppl:S10-S14.

2. [No authors listed]. Global tuberculosis report 2019. World Health Organization. http://www.who.int/tb/publications/global_report/ en/. Published October 17, 2019. Accessed February 19, 2020.

3. Glaziou P, Sismanidis C, Floyd K, Raviglione M. Global epidemiology of tuberculosis. Cold Spring Harb Perspect Med. 2014;5(2):a017798.

4. Houben RM, Dodd PJ. The Global Burden of Latent Tuberculosis Infection: A Re-estimation Using Mathematical Modelling PLoS Med. 2016;13(10):e1002152.

5. Muñoz L, Stagg HR, Abubakar I. Diagnosis and Management of Latent Tuberculosis Infection. Cold Spring Harb Perspect Med. 2015;5(11):a017830

6. Kiazyk S, Ball TB. Latent tuberculosis infection: An overview. Can Commun Dis Rep. 2017;43(3-4):62-66.

7. Restrepo BI, Schlesinger LS. Impact of diabetes on the natural history of tuberculosis. Diabetes Res Clin Pract. 2014;106(2):191-199.

8. Cheekatla SS, et al. NK-CD11c+ Cell Crosstalk in Diabetes Enhances IL-6-Mediated Inflammation during Mycobacterium tuberculosis Infection. PLoS Pathog. 2016;12(10):e1005972.

9. Lopez-Lopez N, et al. Type-2 diabetes alters the basal phenotype of human macrophages and diminishes their capacity to respond, internalise, and control Mycobacterium tuberculosis. Mem Inst Oswaldo Cruz. 2018;113(4):e170326.

10. [No authors listed]. Diabetes facts \& figures. International Diabetes Federation. https://www.idf.org/aboutdiabetes/ what-is-diabetes/facts-figures.html. Accessed February 19, 2020.

11. Restrepo BI. Diabetes and Tuberculosis. Microbiol Spectr. 2016;4(6):10.1128/microbiolspec.TNMI7-0023-2016.

12. Lachmandas E, van den Heuvel CN, Damen MS, Cleophas MC, Netea MG, van Crevel R. Diabetes Mellitus and Increased Tuberculosis Susceptibility: The Role of Short-Chain Fatty Acids. J Diabetes Res. 2016;2016:6014631.

13. Hensel RL, Kempker RR, Tapia J, Oladele A, Blumberg HM, Magee MJ. Increased risk of latent tuberculous infection among persons with pre-diabetes and diabetes mellitus. Int J Tuberc Lung Dis. 2016;20(1):71-78.

14. Lachmandas E, et al. The effect of hyperglycaemia on in vitro cytokine production and macrophage infection with Mycobacterium tuberculosis. PLoS One. 2015;10(2):e0117941.

15. Kumar NP, et al. Type 2 diabetes mellitus coincident with pulmonary tuberculosis is associated with heightened systemic type 1, type 17, and other proinflammatory cytokines. Ann Am Thorac Soc. 2013;10(5):441-449.

16. Tripathi D, et al. IL-22 produced by type 3 innate lymphoid cells (ILC3s) reduces the mortality of type 2 diabetes mellitus (T2DM) mice infected with Mycobacterium tuberculosis. PLoS Pathog. 2019;15(12):e1008140.

17. Jaron B, Maranghi E, Leclerc C, Majlessi L. Effect of attenuation of Treg during BCG immunization on anti-mycobacterial Th1 responses and protection against Mycobacterium tuberculosis. PLoS One. 2008;3(7):e2833.

18. Zumla A, Raviglione M, Hafner R, von Reyn CF. Tuberculosis. N Engl J Med. 2013;368(8):745-755.

19. Boer MC, Prins C, van Meijgaarden KE, van Dissel JT, Ottenhoff TH, Joosten SA. Mycobacterium bovis BCG Vaccination Induces Divergent Proinflammatory or Regulatory T Cell Responses in Adults. Clin Vaccine Immunol. 2015;22(7):778-788.

20. Perdomo C, et al. Mucosal BCG Vaccination Induces Protective Lung-Resident Memory T Cell Populations against Tuberculosis. mBio. 2016;7(6):e01686-16.

21. Moliva JI, Turner J, Torrelles JB. Immune Responses to Bacillus Calmette-Guérin Vaccination: Why Do They Fail to Protect against Mycobacterium tuberculosis? Front Immunol. 2017;8:407.

22. Arts RJW, et al. BCG Vaccination Protects against Experimental Viral Infection in Humans through the Induction of Cytokines 
Associated with Trained Immunity. Cell Host Microbe. 2018;23(1):89-100.e5.

23. Kühtreiber WM, et al. Long-term reduction in hyperglycemia in advanced type 1 diabetes: the value of induced aerobic glycolysis with BCG vaccinations. NPJ Vaccines. 2018;3:23

24. Kristensen I, Aaby P, Jensen H. Routine vaccinations and child survival: follow up study in Guinea-Bissau, West Africa. $B M J$. 2000;321(7274):1435-1438.

25. Hirve S, Bavdekar A, Juvekar S, Benn CS, Nielsen J, Aaby P. Non-specific and sex-differential effects of vaccinations on child survival in rural western India. Vaccine. 2012;30(50):7300-7308.

26. Blok BA, Arts RJ, van Crevel R, Benn CS, Netea MG. Trained innate immunity as underlying mechanism for the long-term, nonspecific effects of vaccines. J Leukoc Biol. 2015;98(3):347-356.

27. Fuge O, Vasdev N, Allchorne P, Green JS. Immunotherapy for bladder cancer. Res Rep Urol. 2015;7:65-79.

28. Liu YY, et al. Bacillus Calmette-Guérin and TLR4 agonist prevent cardiovascular hypertrophy and fibrosis by regulating immune microenvironment. J Immunol. 2008;180(11):7349-7357.

29. Han RF, Pan JG. Can intravesical bacillus Calmette-Guérin reduce recurrence in patients with superficial bladder cancer? A meta-analysis of randomized trials. Urology. 2006;67(6):1216-1223.

30. Kühtreiber WM, Faustman DL. BCG Therapy for Type 1 Diabetes: Restoration of Balanced Immunity and Metabolism. Trends Endocrinol Metab. 2019;30(2):80-92.

31. Punnonen J, Carballido JM, Aversa G, de Vries JE. Interleukin 13 and its Receptor. In: Delves PJ, ed. Encyclopedia of Immunology. Oxford: Elsevier; 1998:1489-1492.

32. Ochoa-Repáraz J, et al. IL-13 production by regulatory T cells protects against experimental autoimmune encephalomyelitis independently of autoantigen. J Immunol. 2008;181(2):954-968.

33. Mohning MP, Downey GP, Cosgrove GP, Redente EF. Chapter 3 - Mechanisms of Fibrosis. In: Swigris JJ, Brown KK, eds. Idiopathic Pulmonary Fibrosis. Oxford, UK;Elsevier; 2019:9-31.

34. Martens GW, Arikan MC, Lee J, Ren F, Greiner D, Kornfeld H. Tuberculosis susceptibility of diabetic mice. Am J Respir Cell Mol Biol. 2007;37(5):518-524.

35. Jeon CY, Murray MB. Diabetes mellitus increases the risk of active tuberculosis: a systematic review of 13 observational studies. PLoS Med. 2008;5(7):e152.

36. Leung CC, et al. Diabetic control and risk of tuberculosis: a cohort study. Am J Epidemiol. 2008;167(12):1486-1494

37. Gan YH. Host susceptibility factors to bacterial infections in type 2 diabetes. PLoS Pathog. 2013;9(12):e1003794.

38. Brosch R, et al. Genome plasticity of BCG and impact on vaccine efficacy. Proc Natl Acad Sci USA. 2007;104(13):5596-5601.

39. Packe GE, Innes JA. Protective effect of BCG vaccination in infant Asians: a case-control study. Arch Dis Child. 1988;63(3):277-281.

40. Black GF, et al. BCG-induced increase in interferon-gamma response to mycobacterial antigens and efficacy of BCG vaccination in Malawi and the UK: two randomised controlled studies. Lancet. 2002;359(9315):1393-1401.

41. Soares AP, et al. Bacillus Calmette-Guérin vaccination of human newborns induces T cells with complex cytokine and phenotypic profiles. J Immunol. 2008;180(5):3569-3577.

42. Lalor MK, et al. Complex cytokine profiles induced by BCG vaccination in UK infants. Vaccine. 2010;28(6):1635-1641.

43. Kagina BM, et al. Delaying BCG vaccination from birth to 10 weeks of age may result in an enhanced memory CD4 T cell response. Vaccine. 2009;27(40):5488-5495.

44. Burl S, et al. Delaying bacillus Calmette-Guérin vaccination from birth to $41 / 2$ months of age reduces postvaccination Th1 and IL-17 responses but leads to comparable mycobacterial responses at 9 months of age. J Immunol. 2010;185(4):2620-2628.

45. Djuardi Y, Sartono E, Wibowo H, Supali T, Yazdanbakhsh M. A longitudinal study of BCG vaccination in early childhood: the development of innate and adaptive immune responses. PLoS One. 2010;5(11):e14066.

46. Dockrell HM. A new challenge for the tuberculosis vaccine community? J Infect Dis. 2012;205(7):1029-1031.

47. Akkoc T, et al. Neonatal BCG vaccination induces IL-10 production by CD4+ CD25+ T cells. Pediatr Allergy Immunol. 2010;21(7):1059-1063.

48. Li L, Qiao D, Zhang X, Liu Z, Wu C. The immune responses of central and effector memory BCG-specific CD4+ T cells in BCG-vaccinated PPD+ donors were modulated by Treg cells. Immunobiology. 2011;216(4):477-484.

49. Boer MC, van Meijgaarden KE, Joosten SA, Ottenhoff TH. CD8+ regulatory T cells, and not CD4+ T cells, dominate suppressive phenotype and function after in vitro live Mycobacterium bovis-BCG activation of human cells. PLoS One. 2014;9(4):e94192.

50. Sakaguchi S, Yamaguchi T, Nomura T, Ono M. Regulatory T cells and immune tolerance. Cell. 2008;133(5):775-787.

51. Sakaguchi S. Regulatory T cells: history and perspective. Methods Mol Biol. 2011;707:3-17.

52. Kursar M, et al. Cutting Edge: Regulatory T cells prevent efficient clearance of Mycobacterium tuberculosis. J Immunol. 2007;178(5):2661-2665.

53. Díaz A, et al. Increased frequency of CD4+ CD25+ FoxP3+ T regulatory cells in pulmonary tuberculosis patients undergoing specific treatment and its relationship with their immune-endocrine profile. J Immunol Res. 2015;2015:985302.

54. Hasegawa $\mathrm{H}$, et al. Therapeutic effect of CXCR3-expressing regulatory $\mathrm{T}$ cells on liver, lung and intestinal damages in a murine acute GVHD model. Gene Ther. 2008;15(3):171-182.

55. Koch MA, Tucker-Heard G, Perdue NR, Killebrew JR, Urdahl KB, Campbell DJ. The transcription factor T-bet controls regulatory $\mathrm{T}$ cell homeostasis and function during type 1 inflammation. Nat Immunol. 2009;10(6):595-602.

56. Sharma MD et al. Indoleamine 2,3-dioxygenase controls conversion of Foxp3+ Tregs to TH17-like cells in tumor-draining lymph nodes. Blood. 2009;113(24):6102-6111.

57. Hoerning A, et al. Subsets of human CD4(+) regulatory T cells express the peripheral homing receptor CXCR3. Eur J Immunol. 2011;41(8):2291-2302.

58. Zhou X, et al. Instability of the transcription factor Foxp3 leads to the generation of pathogenic memory T cells in vivo. Nat Immunol. 2009;10(9):1000-1007.

59. McLeod JJ, Baker B, Ryan JJ. Mast cell production and response to IL-4 and IL-13. Cytokine. 2015;75(1):57-61.

60. Sica A, Schioppa T, Mantovani A, Allavena P. Tumour-associated macrophages are a distinct M2 polarised population promoting 
tumour progression: potential targets of anti-cancer therapy. Eur J Cancer. 2006;42(6):717-727.

61. Atri C, Guerfali FZ, Laouini D. Role of Human Macrophage Polarization in Inflammation during Infectious Diseases. Int J Mol Sci. 2018;19(6):E1801

62. Sheikh F, et al. The Interleukin-13 Receptor- $\alpha 1$ Chain Is Essential for Induction of the Alternative Macrophage Activation Pathway by IL-13 but Not IL-4. J Innate Immun. 2015;7(5):494-505.

63. Proto JD, et al. Regulatory T Cells Promote Macrophage Efferocytosis during Inflammation Resolution. Immunity. 2018;49(4):666-677.e6.

64. Liu Q, et al. IL-33-mediated IL-13 secretion by ST2+ Tregs controls inflammation after lung injury. JCI Insight. 2019;4(6):123919.

65. Bhattacharjee A, Shukla M, Yakubenko VP, Mulya A, Kundu S, Cathcart MK. IL-4 and IL-13 employ discrete signaling pathways for target gene expression in alternatively activated monocytes/macrophages. Free Radic Biol Med. 2013;54:1-16.

66. Martinez FO, Helming L, Gordon S. Alternative activation of macrophages: an immunologic functional perspective. Annu Rev Immunol. 2009;27:451-483.

67. Tundup S, Srivastava L, Harn DA. Polarization of host immune responses by helminth-expressed glycans. Ann N Y Acad Sci. 2012;1253:E1-E13

68. Nair MG, Herbert DR. Immune polarization by hookworms: taking cues from T helper type 2, type 2 innate lymphoid cells and alternatively activated macrophages. Immunology. 2016;148(2):115-124.

69. Mège JL, Mehraj V, Capo C. Macrophage polarization and bacterial infections. Curr Opin Infect Dis. 2011;24(3):230-234

70. Patel SK, Beaino W, Anderson CJ, Janjic JM. Theranostic nanoemulsions for macrophage COX-2 inhibition in a murine inflammation model. Clin Immunol. 2015;160(1):59-70.

71. Weiss G, Schaible UE. Macrophage defense mechanisms against intracellular bacteria. Immunol Rev. 2015;264(1):182-203

72. Gordon S, Martinez FO. Alternative activation of macrophages: mechanism and functions. Immunity. 2010;32(5):593-604.

73. Parisi L, et al. Macrophage Polarization in Chronic Inflammatory Diseases: Killers or Builders? J Immunol Res. 2018;2018:8917804

74. Saradna A, Do DC, Kumar S, Fu QL, Gao P. Macrophage polarization and allergic asthma. Transl Res. 2018;191:1-14.

75. Benoit M, Desnues B, Mege JL. Macrophage polarization in bacterial infections. J Immunol. 2008;181(6):3733-3739.

76. Murray PJ, Wynn TA. Protective and pathogenic functions of macrophage subsets. Nat Rev Immunol. 2011;11(11):723-737.

77. Bai X, et al. Inhibition of nuclear factor-kappa B activation decreases survival of Mycobacterium tuberculosis in human macrophages. PLoS One. 2013;8(4):e61925.

78. Kaufmann SHE, Dorhoi A. Molecular Determinants in Phagocyte-Bacteria Interactions. Immunity. 2016;44(3):476-491.

79. Kanter JE, et al. Diabetes promotes an inflammatory macrophage phenotype and atherosclerosis through acyl-CoA synthetase 1 . Proc Natl Acad Sci U S A. 2012;109(12):E715-E724.

80. Kraakman MJ, Murphy AJ, Jandeleit-Dahm K, Kammoun HL. Macrophage polarization in obesity and type 2 diabetes: weighing down our understanding of macrophage function? Front Immunol. 2014;5:470.

81. Dhiman R, et al. NK1.1+ cells and IL-22 regulate vaccine-induced protective immunity against challenge with Mycobacterium tuberculosis. J Immunol. 2012;189(2):897-905.

82. Venkatasubramanian S, et al. A rho GDP dissociation inhibitor produced by apoptotic T-cells inhibits growth of Mycobacterium tuberculosis. PLoS Pathog. 2015;11(2):e1004617.

83. Tripathi D, et al. Alcohol enhances type 1 interferon- $\alpha$ production and mortality in young mice infected with Mycobacterium tuberculosis. PLoS Pathog. 2018;14(8):e1007174.

84. Tripathi D, et al. c-Jun N-terminal kinase 1 defective CD4+CD25+FoxP3+ cells prolong islet allograft survival in diabetic mice. Sci Rep. 2018;8(1):3310.

85. Sibila M, Aragón V, Fraile L, Segalés J. Comparison of four lung scoring systems for the assessment of the pathological outcomes derived from Actinobacillus pleuropneumoniae experimental infections. BMC Vet Res. 2014;10:165. 